LAWRENCE LIVERMORE N A T IO N A L LABORATORY
Computational Prediction of Aerodynamic Forces for a Simplified Integrated Tractor-Trailer Geometry

K. Salari, J.M. Ortega, P.J. Castellucci

June 24, 2004

AIAA Fluid Dynamics Meeting

Portland, OR, United States

June 28, 2004 through July 1, 2004 
This document was prepared as an account of work sponsored by an agency of the United States Government. Neither the United States Government nor the University of California nor any of their employees, makes any warranty, express or implied, or assumes any legal liability or responsibility for the accuracy, completeness, or usefulness of any information, apparatus, product, or process disclosed, or represents that its use would not infringe privately owned rights. Reference herein to any specific commercial product, process, or service by trade name, trademark, manufacturer, or otherwise, does not necessarily constitute or imply its endorsement, recommendation, or favoring by the United States Government or the University of California. The views and opinions of authors expressed herein do not necessarily state or reflect those of the United States Government or the University of California, and shall not be used for advertising or product endorsement purposes.

This work was performed under the auspices of the U. S. Department of Energy by the University of California, Lawrence Livermore National Laboratory under Contract No. W-7405-Eng-48. 


\section{Computational Prediction of Aerodynamic Forces for a Simplified Integrated Tractor-Trailer Geometry}

Kambiz Salari ${ }^{1}$, Jason M. Ortega ${ }^{2}$, and Paul J. Castellucci ${ }^{3}$.

Lawrence Livermore National Laboratory, Livermore, CA 94551

\section{ABSTRACT}

${ }^{1}$ Staff Scientist, Computational Physics Group / NTED, P.O. Box 808, L-644, and Associate Fellow.

${ }^{2}$ Staff Scientist, Computational Physics Group / NTED, P.O. Box 808, L-644, and Member.

${ }^{3}$ Student, Institute for Scientific Computing Research, P.O. Box 808, L-644, and Member. 


\section{INTRODUCTION}

A typical class 8 tractor-trailer traveling at highway speed requires approximately $65 \%$ of the total energy produced by the engine to overcome aerodynamic drag. It is estimated that reducing the drag coefficient of a heavy vehicle by $25 \%$ would result in a total yearly savings of roughly 2 billion gallons of diesel fuel. ${ }^{1}$ In 1996, the U.S. Department of Energy Office of Heavy Vehicle Technologies (OHVT) recognized this potential benefit and founded a research and development effort to reduce the aerodynamic drag/emissions of heavy vehicles. The purpose of this effort has been to identify and to establish through use of experiments and computational simulations the dominant flow structures around heavy vehicle that are responsible for significant contribution to the total aerodynamic drag. Such flow structures exist in the gap between the tractor and the trailer, the vehicle underbody, and the base of the trailer. ${ }^{2}$ Given the knowledge gained in this process, drag reducing devices could be designed to mitigate the effects of these flow structures and, thereby, reduce the aerodynamic drag of tractor-trailers.

This DOE research project is investigating the applicability of the state-of-the-art computational modeling and simulations to predict the flow field surrounding bluff bodies. This serves to provide expertise in applying flow modeling tools in the aerodynamic design process of heavy vehicles. Contributors to this program include seven organizations:! Argonne National Laboratory (ANL), California Institute of Technology (Caltech), Georgia Tech Research Institute (GTRI), Lawrence Livermore National Laboratory (LLNL), National Aeronautics and Space Administration (NASA), Sandia National Laboratories (SNL), and University of Southern California (USC).

Traditionally, heavy vehicle aerodynamicists have relied upon experimental data from wind tunnels and road tests for design purposes. However, computer simulations of aerodynamic flow around heavy vehicles have significantly matured over the past five years. Thus, truck manufacturers are beginning to integrate this new capability into their vehicle aerodynamic design process. LLNL's responsibility in this effort is to provide heavy vehicle manufacturers with guidance on computational modeling and simulations, advanced numerical schemes, grid generation, turbulence modeling, solution accuracy, and also aero-devices for drag reduction. The truck industry heavily depends on steady Reynolds-Averaged Navier-Stokes (RANS) computations (commercial codes) to study the drag of heavy vehicles. This investigation will highlight the strengths and the weaknesses of RANS in predicting the flow field and the resulting aerodynamic forces for a simplified heavy vehicle. We conduct steady computations using RANS on a generic tractor-trailer type geometry known as Ground Transportation System or GTS designed by Sandia National Laboratories. Gutierrez et al. ${ }^{3}$ gives an overview of the ground transportation system project and also provides the details of the GTS geometry. The GTS geometry has been tested in the Texas A\&M University 7' $\square 10^{\prime}$ wind tunnel in $1995^{4}$ and in the NASA Ames 7' $\square 10^{\prime}$ wind tunnel in $1999^{5}$. Computational flow simulations have also been performed on the GTS geometry. ${ }^{6-8}$ 
The layout of the remainder of the paper is as follows. The criteria for these validation simulations are described in Section ?.!!!!!!!!!!!!

Particular attention is given to the validation simulations, which are discussed in the next section.

\section{NASA AMES WIND TUNNEL EXPERIMENT}

The $1 / 8^{\text {th }}$-scale GTS model was studied experimentally in the NASA Ames 7 ' $110^{\prime}$ wind tunnel in $1999 .^{5}$ Experimental measurements were made of aerodynamic forces, surface pressures, surface skin friction (oil film interferometry), and 3-D velocity fields (particle image velocimetry, PIV). Figure 1 shows the GTS model installation in NASA Ames $7^{\prime} \square^{\prime} 10^{\prime}$ wind tunnel. The GTS model was instrumented with a total of 79 surface pressure taps as shown in Figure 2. An additional 60 surface pressure taps are positioned on the tunnel wall as shown in Figure 3. Among the wall surface pressure taps a reference location is chosen to be used in calculation of pressure coefficients. A tabular list of the tap locations is available from Storms et al. ${ }^{5}$

The surface pressure coefficient $C_{p}$ was computed using the wall reference pressure $P_{s w}$ and the tunnel dynamic pressure, $q_{\text {. }}$, as follows.

$$
C_{p}=\frac{p \square p_{s w}}{q}
$$

where q. was computed from the difference between the settling-chamber static pressure, $p_{t}$, and the reference tap wall pressure.

Instantaneous and time averaged flow field in the wake of the trailer is provided by the three-component PIV for several laser light sheet plane orientations as shown in Table 1. The coordinates in this table are non-dimensionalized by the width of the trailer $w=12.75$ in $(32.38 \mathrm{~cm})$. The empty tunnel test-section turbulence intensity is $0.25 \%$ at a Mach number, $\mathrm{M}$, of 0.22 .

The uncertainties in the measured and computed parameters are: $\pm 0.1^{\circ}$ for the yaw angle ${ }^{9} ; \pm 0.01$ for the aerodynamic force coefficients; \pm 0.004 for the computed 
pressure coefficients ${ }^{9} ; \pm 2 \%$ for in-plane PIV velocities; and $\pm 4 \%$ for out-of-plane PIV velocities.

\begin{tabular}{|c|l|l|l|}
\hline Orientation & \multicolumn{1}{|c|}{ Location } & \multicolumn{1}{c|}{ Yaw } & \multicolumn{1}{c|}{$\mathbf{R e} / 10^{6}$} \\
\hline Horizontal & $\mathrm{y} / \mathrm{w}=0.35,0.70,1.05$ & $0^{\circ}, 10^{\circ}$ & $2.0,0.74,0.3$ \\
\hline Cross-stream & $\mathrm{x} / \mathrm{w}=8.00,8.35,8.78$ & $0^{\circ}, 10^{\circ}$ & $2.0,0.74$ \\
\hline Streamwise & $\mathrm{z} / \mathrm{w}=0.0$ & $0^{\circ}$ & $2.0,0.74$ \\
\hline
\end{tabular}

Table 1: Laser sheet orientations. ${ }^{5}$

\section{WIND TUNNEL MODELING}

To accurately capture the flow condition in the tunnel test-section, a portion of the NASA Ames 7' 10 ' wind tunnel was modeled. The locations of the inflow and outflow boundaries determined what portion of the tunnel was modeled. Since the GTS model was mounted on the tunnel floor and was exposed to the floor boundary layer, the accuracy of a computational prediction depended on how well the upstream boundary layer profile was represented. To capture the proper boundary layer profile and mass flow rate at the test-section entrance, the inflow boundary was positioned far upstream from the test-section. The outflow boundary was positioned downstream of the test-section in the diffuser to prevent interference with the trailer wake flow structure. The initial computational domain (Figure 4a) contained the settling chamber, contraction, test-section and part of expansion of the $7^{\prime} \square^{\prime} 0^{\prime}$ wind tunnel. However, after several computations, it was decided that a truncated wind tunnel model (Figure 4b) was sufficient to accurately represent the flow in the test-section, including the incoming boundary layer.

Modeling a portion of the wind tunnel allowed use of :

- wind tunnel data not corrected for model blockage

- the experimental reference pressure location in the calculation of pressure coefficients

- the experimental boundary layer measurements

The truncated wind tunnel geometry was used for all subsequent simulations. Since the model is significantly far from the top and side walls, they were modeled as slip boundaries, while the tunnel floor was modeled as a no-slip boundary. For the inflow boundary, the total conditions (pressure and temperature) were held constant and the velocity field was allowed to develop. For the outflow boundary, a static pressure was defined. Some of the required parameters for inflow and outflow boundaries are 
not available from the experiment and needed to be evaluated. Details on how to obtain these parameters are provided in Section ???.

\section{COMPUTATIONAL TOOLS AND POST-PROCESSING}

NASA OVERFLOW v2.0s, which is a fully compressible, 3-D, finite volume code employing overset grids, was used for all simulations. The pre-processing necessary for solution of overset grids in OVERFLOW was achieved with PEGASUS v5. This code prepares volume grids for the flow solver by computing the interpolation stencil and removing grid points, which are contained inside solid bodies. Forces and moments on the overset surface grids were computed by FOMOCO. Interactive Data Language (IDL), Ensight (Computational Engineering International), and Tecplot (Amtec Engineering Inc.) were used in post-processing and feature extraction.

\section{TURBULENCE MODEL SELECTION}

One of the goals of the DOE research initiative is to provide the tractor and the trailer manufacturers with a knowledge base that describes the advantages and disadvantages of commonly used turbulence models in predicting heavy vehicle aerodynamics. The following commonly used RANS turbulence models were selected: one-equation Spalart-Allmaras (SA), two-equation Wilcox k- $\mathrm{C}, 1988$ version (KW), and two-equation Mentor SST models (SST). The Mentor SST model was used in the baseline mode in which the shear-stress limiter was turned off. In all simulations, the flow was assumed to be fully turbulent and transition was not modeled. Also, all turbulence equations were integrated to the wall and no wall functions were used. We realized that wall function usage is prevalent in commercial codes and can potentially reduce the number of elements needed in the simulation by $50 \%$. Argonne National Laboratory, as part of the DOE consortium, is currently investigating the influence of wall functions on predicting the flow field around heavy vehicles.

The type of discretization techniques that are applied to the turbulence equations can influence the predictive capability of these models. In most commercial codes, the advective part of these equations are typically discretized using the first-order upwind scheme to ensure robustness. However, this scheme is quite dissipative and can influence the computational results. In this effort, a sensitivity study was performed on the first-order vs. the second-order treatment of the advective part of the turbulence equations. 


\section{MESH GENERATION}

GRIDGEN was used to construct all meshes needed for this investigation. For the empty tunnel simulations, two single-block grids (Figure 5) with 466,240 and 816,000 elements were constructed. For the GTS baseline model in the NASA Ames 7' $\square 10^{\prime}$ wind tunnel, two grids for each yaw angle $\left(0^{\circ}\right.$ and $\left.10^{\circ}\right)$ were generated. Grid statistics for these meshes are presented in Tables 2 and 3. For all no-slip surfaces, y+ values of about 0.4-0.5 are maintained for all grids. All meshes are non-dimensionalized by the GTS trailer width ( $w=12.75$ in or $32.38 \mathrm{~cm})$.

\begin{tabular}{|c|c|c|c|c|}
\hline Yaw angle & No. of elements & $\begin{array}{c}\text { Min spacing in } \\
\square\end{array}$ & $\begin{array}{c}\text { Min spacing in } \\
\square\end{array}$ & $\begin{array}{c}\text { Min spacing in } \\
\square\end{array}$ \\
\hline $0^{\circ}$ yaw & $11.8 \mathrm{M}$ & $0.20 \mathrm{~mm}$ & $0.20 \mathrm{~mm}$ & $0.20 \mathrm{~mm}$ \\
\hline $10^{\circ}$ yaw & $14.0 \mathrm{M}$ & $0.20 \mathrm{~mm}$ & $0.20 \mathrm{~mm}$ & $0.20 \mathrm{~mm}$ \\
\hline
\end{tabular}

Table 2: Coarse mesh statistics for 0 and 10 yaw

\begin{tabular}{|c|c|c|c|c|}
\hline Yaw angle & No. of elements & Min spacing in & Min spacing in & Min spacing in \\
\hline $0^{\circ}$ yaw & $14.0 \mathrm{M}$ & $0.19 \mathrm{~mm}$ & $0.19 \mathrm{~mm}$ & $0.19 \mathrm{~mm}$ \\
\hline $10^{\circ}$ yaw & $19.0 \mathrm{M}$ & $0.19 \mathrm{~mm}$ & $0.19 \mathrm{~mm}$ & $0.19 \mathrm{~mm}$ \\
\hline
\end{tabular}

Table 3: Medium mesh statistics for 0 and 10 yaw

The origin of the mesh coordinate system is located on the tunnel floor at the entrance of the test-section along the symmetry plane, which is different from that given in the NASA TM report ${ }^{5}$ (Figure 5). All computed results are presented in the mesh coordinate system.

\section{COMPUTATIONAL SETUP}

Subsonic GTS computations were run using the low-Mach number preconditioner in OVERFLOW. The ARC3D, 3-factor, diagonal scheme was used with a $4^{\text {th }}$-order dissipation term that was set to 0.01 for stability. To accelerate convergence toward steady-state, grid sequencing and Jacobian time-step scaling was employed. All computations were performed using 256 processors on a tightly coupled Linux cluster (11.2 TF) with 1,152 nodes, each with two 2.4-GHz Pentium 4 Xeon processors and 
4 GB of memory. Typical simulation time is about 24 hours for the medium-sized meshes.

\section{TUNNEL EMPTY SIMULATIONS}

Empty tunnel simulations were needed to establish proper boundary layer profile at the entrance to the test-section. Run 404 from the NASA experiment ${ }^{5}$ was selected for empty tunnel simulations. The following parameters, at the tunnel pressure ring are given for this run: total pressure $97984.845 \mathrm{n} / \mathrm{m}^{2}$, total temperature $291.827^{\circ} \mathrm{K}$, and static pressure $94558.03 \mathrm{~N} / \mathrm{m}^{2}$. In the NASA experiment, the boundary layer profile was measured by a boundary layer rake at the origin of the mesh coordinate system. Figure 5 shows the size of the computational domain. The inflow boundary condition maintains the total pressure and temperature by extrapolating the mass flow. The tunnel mass flow was computed at the pressure ring location in the contraction section at $\mathrm{x} / \mathrm{w}=-7.784$ from

$$
\dot{m}=\frac{P_{t} A M \sqrt{\square}}{\sqrt{R T_{t}}} g^{1}+\frac{\square \square}{2} M^{2} 9^{\frac{\square+1}{2 \square \square}}
$$

where $A=6.5032 \mathrm{~m}^{2}$ is the cross-sectional area of the test-section and $M$ is the average Mach number at the test-section computed by

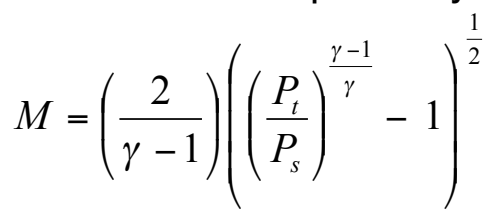

The test-section Mach number from Eq. 3 was computed to be 0.226 . Substituting this value into Eq. 2, we obtained the empty tunnel mass flow rate of $571.26 \mathrm{~kg} / \mathrm{s}$. Next, the inflow Mach number was computed from Eq. 2 knowing the mass flow rate and the inflow area $A=9.2514 \mathrm{~m}^{2}$. The solution to Eq. 3 has two real parts. The subsonic solution 0.156 was picked to be the inflow Mach number. Given this Mach number, the inflow static pressure was computed to be $94,558.03 \mathrm{~N} / \mathrm{m}^{2}$ and static temperature to be $291.73^{\circ} \mathrm{K}$. Two grids were constructed for empty tunnel simulations with 466,240 and 816,000 elements, which are labeled as coarse and medium meshes, respectively.

The specified pressure outflow boundary condition requires the ratio of inflow to outflow static pressures. Multiple empty tunnel simulations were performed to match the Mach number at the test-section mid-height above the center of the turntable to that of the experiments by adjusting the outflow static pressure. After five to six iterations, we obtained an outflow pressure ratio of 0.9992 , which matched the 
experimental test-section Mach number to within $0.07 \%$. This back pressure was then held constant for both the coarse and medium mesh calculations.

Figure 8 presents Mach contours on the tunnel symmetry plane. This figure highlights the uniformity of the velocity field in the test-section and also the growing boundary layer on the tunnel floor. Figure 9 shows the pressure contours on the same plane. As expected the pressure is also uniform in the test-section. Figure 10 presents the comparison of the computed boundary layer profile at the entrance to the test-section (symmetry plane) to the measured NASA experimental data. All turbulence models performed reasonably well in predicting the correct boundary profile for the testsection. As was stated earlier, the shape of this boundary layer profile will impact the accuracy of GTS flow calculations. Figure 11 presents similar calculations with only Mentor SST model using coarse and medium meshes. There is virtually no difference in the profile shape which suggests we have a grid resolved solution for the empty tunnel simulation.

For the GTS flow simulations, which are presented in the following sections, the tunnel resolution is increased in the vicinity of the GTS model to aid the transfer of information from the tunnel grid to the overlapping GTS grids.

\section{COMPUTATIONAL RESULTS}

NASA Ames wind tunnel test provides data for range of yaw angles from $-14^{\circ}$ to $+14^{\circ}$. Among these yaw angles $0^{\circ}$ and $10^{\circ}$ are selected to be studied. The $0^{\circ}$ yaw provides the baseline case in which the flow remains attached over the GTS body except for the base of the trailer. This is the ideal case for the selected turbulence models to perform well. However, at $10^{\circ}$ yaw there are multiple separation regions around the GTS body along with multiple vortex structures. For the $0^{\circ}$ yaw the pressure distribution on the body is compared to the pressure tap data, the wake flow structure is compared to the time averaged PIV data, and the aerodynamic forces are compared to the force data. For the $10^{\circ}$ yaw, the oil-film interferometry images on the top of the trailer are compared to the computed results in addition to the comparisons mentioned for the $0^{\circ}$ yaw.

To investigate the influence of the $1^{\text {st }}$ - versus the $2^{\text {nd }}$-order discretization of the advective part of the turbulence equations, additional simulations are performed on the coarse and the medium mesh at $10^{\circ}$ yaw using the Mentor SST (BSL) model.

The trailer wake flow structure is investigated by comparing the computed results to the time-averaged PIV data from NASA experiment. The available PIV laser light sheet planes includes the streamwise vertical plane at $(\mathrm{y} / \mathrm{w}=0)$ for $0^{\circ}$ yaw, the horizontal plane at the trailer mid-height for $0^{\circ}$ and $10^{\circ}$ yaw, the cross-stream plane at distances of $4.5 / 12.75$ " and $14.5 / 12.75 "$ for $0^{\circ}$ and $10^{\circ}$ yaw, respectively. Note that 
the PIV laser sheet plane coordinate system for the $0^{\circ}$ and $10^{\circ}$ yaw are different. At $0^{\circ}$ yaw, the data are presented with a body fixed coordinate system as shown in figure 2. At $10^{\circ}$ yaw, the coordinate system remains aligned with GTS, but the origin lies afore and starboard of the model. To highlight the location of the laser light sheet its position is included in relevant particle traces plots. For all particle trace plots showing the computed flow structure in the wake of the trailer identical particle emitters are used.

Tunnel-aligned velocity components were extracted along the GTS model centerline for comparison with the PIV data set for $0^{\circ}$ and $10^{\circ}$ yaw. There is some disagreement among PIV sheets for centerline tunnel-axis velocities beyond the $2 \%$ to $4 \%$ quoted errors. This may be due to the fact that different freestream values used to normalize data from sheets of different orientation.

In the next section detailed results for each yaw angle is presented.

\section{RESULTS FOR $0^{\circ}$ YAW}

From NASA experiment run 7 point 9 with GTS baseline configuration at $0^{\circ}$ is selected. The experimental error for setting the yaw angle is about $\pm 0.1^{\circ}$. The following flow condition is provided for this run: Mach number 0.28 , velocity 93.91 $\mathrm{m} / \mathrm{s}$, dynamic pressure $5314.89 \mathrm{~N} / \mathrm{m}^{2}$, Reynolds number $2.08 \times 10^{6}$ (based on trailer width), total pressure $102649.217 \mathrm{~N} / \mathrm{m}^{2}$, total temperature $284.66^{\circ} \mathrm{K}$, static pressure $97339.115 \mathrm{~N} / \mathrm{m}^{2}$, and static temperature $280.36^{\circ} \mathrm{K}$, and air density $1.206 \mathrm{~kg} / \mathrm{m}^{3}$.

Two grids are generated for this case labeled as coarse and medium mesh. The statistics for these meshes are presented in tables 2 and 3.

The tunnel mass flow rate of $714.191 \mathrm{~kg} / \mathrm{s}$ was computed at the tunnel pressure ring with the static pressure of $98333.732 \mathrm{~N} / \mathrm{m}^{2}$ using equations 3 and 4 . Knowing the mass flow rate, the inflow Mach number of 0.1854 was computed from equation 3 . Similar procedure as outlined in empty tunnel section is followed to compute the inflow Mach number. Hence, the inflow parameters are: Mach 0.1854 , static pressure $100,214.496 \mathrm{~N} / \mathrm{m}^{2}$, static temperature $282.715^{\circ} \mathrm{K}$, and inflow Re $1.42 \times 10^{6}$.

For the outflow static pressure multiple runs were performed, with medium grid and Mentor SST turbulence model, by varying the outflow static pressure to match the the computed pressure ring Mach number. The outflow pressure ratio (outflow/inflow) of 0.99822 was obtained that matched the pressure ring Mach number within $0.08 \%$. This suggests the test-section flow condition is reasonably represented and we can proceed with GTS simulations.

The following simulations are performed at $0^{\circ}$ yaw, 


\begin{tabular}{|c|c|c|c|}
\hline Mesh & \multicolumn{3}{|c|}{ Turbulence Model } \\
\hline & Mentor SST & Wilcox k-w & SA \\
\hline Medium & $\mathrm{x}$ & $\mathrm{X}$ & $\mathrm{X}$ \\
\hline Coarse & $\mathrm{x}$ & & \\
\hline
\end{tabular}

Table 4: Run matrix for the $0^{\circ}$ yaw

The initial result from the Mentor SST turbulence model with the medium mesh produced a non-physical separation in front of the GTS on one side close to the midheight around the sharp radii of the tractor. The separation region was unaffected by grid sequence and mesh resolution. The shear stress limiting of the SST model was identified as a possible cause of the separation. The overflow code was modified to turn the limiter off. The new simulations with no active limiter showed no sign of the separation region. Hence, for all simulations the Mentor SST turbulence model was used with no shear stress limiting (baseline mode).

For all simulations the residuals were reduced to machine zero. Figures $x x$ and $x x$ show a typical convergence history and drag force coefficient versus number of iterations for the medium grid SST baseline turbulence model. ( need figures ????)

Figure 12 present medium grid SST (BSL) solution using particle traces that are colored by velocity magnitude around the GTS model. This figure shows a relatively simple flow structure with no massive flow separation or vortex structures except for the wake of the trailer. Figures 13-15 presents the flow field around the GTS model. Figure 13 shows the velocity magnitude contours at the symmetry plane $(y / w=0)$ around the GTS geometry. This figure highlights the boundary layer growth on top of the trailer and the velocity field around the tractor and under the GTS model. It also shows the flow accelerating between the four supporting posts. Figure 14 shows the velocity field on the horizontal cut-plane at $(\mathrm{z} / \mathrm{w}=$ ??). The boundary layer growth on the side of the GTS and the tractor flow field is clearly visible. Figure 15 presents the velocity field on the vertical cut-plane $(\mathrm{x} / \mathrm{w}=$ ?). This figure presents the flow field around the trailer close to the base, the influence of the support, and the tunnel floor boundary layer. At this yaw angle all turbulence models produced similar flow field as depicted in figures 12-15 except for the wake.

Table 5 presents the computed aerodynamic force coefficients including the pressure and viscous contribution for the selected turbulence models compared to the NASA experimental data. Among the selected models Mentor SST (BSL) predicted the closest drag to the experimental data. The medium mesh result is within $0.3 \%$ and the coarse mesh result is within $2 \%$ of the experimental data, which has $3.8 \%$ uncertainty. The Wilcox k-w turbulence model under predicated the drag by about $10 \%$ and the SA model over predicated the drag by about $21 \%$. For the lift coefficient all models predicted a similar result with the difference ranging from $33 \%$ for Wilcox $\mathrm{k}$-w model to about $18 \%$ for the SA model. The uncertainty on the experimental lift coefficient is about $6.2 \%$. All models predicted the side force within the experimental 
uncertainty range of $133 \%$. The SST (BSL) model produced similar result for the lift and the side force on the medium and coarse mesh. To investigate why there is so much difference in drag prediction among the turbulence models, lets start with examining the pressure field around the GTS model.

\begin{tabular}{|c|c|c|c|c|c|c|c|}
\hline Medium grid, SST & & CD & Error & CL & Error & CS & Error \\
\hline & Pressure & 0.1897 & & -0.1226 & & $-1.4 \mathrm{E}-05$ & \\
\hline & Viscous & 0.0741 & & 0.0012 & & $-1.5 \mathrm{E}-07$ & \\
\hline & Total & 0.2638 & $0.3 \%$ & -0.1214 & $24.1 \%$ & $-1.4 \mathrm{E}-05$ & $-100 \%$ \\
\hline \multirow[t]{4}{*}{ Medium grid, KW } & & CD & & CL & & CS & \\
\hline & Pressure & 0.1603 & & -0.1083 & & $-4.3 \mathrm{E}-04$ & \\
\hline & Viscous & 0.0775 & & 0.0013 & & $-8.1 E-07$ & \\
\hline & Total & 0.2377 & $-9.6 \%$ & -0.1070 & $33.1 \%$ & $-4.3 \mathrm{E}-04$ & $-106 \%$ \\
\hline \multirow[t]{4}{*}{ Medium grid, SA } & & CD & & CL & & CS & \\
\hline & Pressure & 0.2435 & & -0.1329 & & 5.3E-05 & \\
\hline & Viscous & 0.0737 & & 0.0012 & & $-2.3 E-06$ & \\
\hline & Total & 0.3173 & $20.6 \%$ & -0.1317 & $17.7 \%$ & 5.1E-06 & $-99 \%$ \\
\hline \multirow[t]{4}{*}{ Coarse grid, SST } & & CD & & CL & & CS & \\
\hline & Pressure & 0.1943 & & -0.1232 & & $-1.1 \mathrm{E}-06$ & \\
\hline & Viscous & 0.0741 & & 0.0012 & & $-1.9 \mathrm{E}-06$ & \\
\hline & Total & 0.2684 & $2.0 \%$ & -0.1220 & $23.7 \%$ & $-3.0 \mathrm{E}-06$ & $-100 \%$ \\
\hline \multirow[t]{4}{*}{$\begin{array}{c}\text { NASA experiment } \\
\pm 0.01 \text { error }\end{array}$} & & CD & & CL & & CS & \\
\hline & Point 2 & 0.263 & $\pm 3.8 \%$ & -0.168 & $\pm 6.2 \%$ & 0.008 & $\pm 133 \%$ \\
\hline & Point 9 & 0.263 & & -0.152 & & 0.007 & \\
\hline & Average & 0.263 & & -0.16 & & 0.0075 & \\
\hline
\end{tabular}

Table 5: Computed aerodynamic forces for the GTS model at $0^{\circ}$ yaw including the NASA experimental data

Figure 16-20 presents the comparison of the computed pressure coefficients, for all turbulence models, to the pressure tap data of NASA Ames. The uncertainty for the 
computed $\mathrm{C}_{\mathrm{p}}$ from the experiment is about 0.004 [4]. This uncertainty is not shown as the error bar because it size is similar to the symbols used in these figures. Figure 16 compares the data of the 3 pressure taps in front of GTS (stagnation area) to the computed solution and the the agreement is reasonable. Figures 17 and 18 present the comparison of pressure coefficient distribution, on the top and the bottom of GTS, to the experimental data. Again the agreement is reasonable except for the top of the trailer very close to the base. This is a critical area before the shear layer moves to the wake and influences the wake flow structure. Hence, to capture the wake flow structure the pressure field in this area should accurately be captured. Figure 19 shows the computed $C_{p}$, on the side of GTS on the mid-section of the trailer, compared to the experimental data. The agreement is slightly off at the beginning of the trailer and it improves toward the base. The disagreement could have different potential causes including the slight deviation in the yaw angle.

Figure 20 presents the comparison of $C_{p}$ to the experimental data at the base of the trailer. It is very clear that all turbulence models failed to capture the pressure field including the trend. Figure 21 shows the comparison of the computed $\mathrm{C}_{\mathrm{p}}$ from the symmetry plane $(\mathrm{y} / \mathrm{w}=0)$ toward the edge of the trailer to all the available pressure taps data on the base of the trailer. The computed results failed to capture the right trend and the magnitude of pressure field on the entire surface of the base of the trailer. This wide variation in the pressure field is the reason behind such as range in drag predictions.

Overall, all turbulence models adequately predicated the pressure field on the body of GTS geometry except on the base of the trailer.

To investigate the mesh resolution influence on the pressure field a coarse mesh simulation is performed using the SST (BSL) turbulence model. Figures 22-24 present grid convergence study for SST baseline turbulence model at the $0^{\circ}$ yaw. Both coarse and medium grids produced a similar solution around the GTS except for the base of the trailer where there is a slight difference in the solution. This suggested that we have a grid independent pressure field.

To better understand the trailer wake flow structure, let's compare the computed solutions to the time-averaged PIV data. The horizontal laser light sheet PIV data at the trailer mid-height (Fig 25) shows the existence of two dominant counter-rotating vortices. All three turbulence models, shown in Figures 26-28, captured these vortices at different axial and spanwise locations. The SA model predicted the vortex core location to be $50 \%$ too close to the base of the trailer, consistent with a smaller wake and $20 \%$ too close together when compared to PIV. The SST (BSL) model fares somewhat better at $20 \%$ in both directions. In contrast, $\mathrm{k}-\mathrm{w}$ model overpredicted the vortex core location and spacing by $10 \%$ and $5 \%$, respectively.

Figure 29 presents particle traces using the PIV data at the cross-stream plane $(x / w=?$ ? $)$. It is difficult to ascertain the dominant flow features in this PIV plane. The 
predictions of all the three turbulence models show the greatest difference (Figs 3032 ) in this cut-plane. The SST (BSL) model does not display any vortex like structure in this plane. The k-w model shows a single, peculiar, vortex away from the model centerline. The SA model displays four distinct vortices in this plane.

Figure 33 presents particle traces for the PIV data at the streamwise cut-plane $(y / w=0)$. None of the turbulence models predicts (Figs 34-36) the behavior shown in PIV data, namely the presence of a single vortex in the lower half of the sheet. In addition, KW is the only model that exhibits any significant vertical asymmetry. This particular cut-plane clearly illustrates the disparity in recirculation zone size among the turbulence models.

To further investigate the trailer wake flow structure, tunnel-aligned velocity components were extracted along the GTS model centerline for all computed solutions, as shown in Figure 37, for comparison to the PIV data set. There is some disagreement among PIV sheets for centerline tunnel-axis velocities which may be due to the fact that different free-stream values used to normalize data from sheets of different orientation. Following the data set documentation, freestream velocities were obtained from zones within each simulation. For the $0^{\circ}$ yaw velocities were normalized by non-dimensional velocities .294. The three turbulence models consistently over-predict the velocity magnitude in the recirculation region. Contrary to intuition, the model that predicts the smallest recirulation zone, SA, does not predict the largest recirculation velocity. The SST model predicts a centerline recirculation velocity of $36 \%$ of freestream, slighty faster than SA, and $20 \%$ larger than KW.

\section{RESULTS FOR $10^{\circ}$ YAW}

For this yaw angle run 7 point 5 with baseline configuration is selected. The same flow condition including the inflow and outflow boundary conditions as $0^{\circ}$ yaw is maintained. Two grids are generated for this case labeled as coarse and medium mesh. The statistics for these meshes are presented in tables 2 and 3 . Since the outflow static pressure is maintained from the $0^{\circ}$ yaw, we anticipate the mean Mach number at the tunnel pressure ring to reduce slightly due to increase blockage. For this yaw angle the computed Mach number at the pressure ring location is within $0.5 \%$ of the experimental value.

The following simulations are performed at $10^{\circ}$ yaw,

\begin{tabular}{|c|c|c|c|c|}
\hline Mesh & \multicolumn{4}{|c|}{ Turbulence Model } \\
\hline & Mentor SST, $1^{\text {st }}$-order & Mentor SST, $2^{\text {nd }}$-order & Wilcox k-w & SA \\
\hline Medium & $\mathrm{x}$ & $\mathrm{x}$ & $\mathrm{x}$ & $\mathrm{x}$ \\
\hline Coarse & $\mathrm{x}$ & $\mathrm{x}$ & & \\
\hline
\end{tabular}


Table 6: Run matrix for the $10^{\circ}$ yaw

For all simulations the residuals were reduced close to machine zero. Figures 37 and 38 show a typical convergence history and drag force coefficient versus number of iterations for the SST baseline turbulence model on the medium grid. (need figures ????)

Figure 39 present the SST (BSL) solution on the medium grid using particle traces that are colored by velocity magnitude. This figure shows a complex flow structure with multiple flow separations and vortex structures around the GTS model. Figures 40 shows the velocity field using the Mach contours at the horizontal cut-plane trailer mid-height. In this figure the separation bubble on the lee-ward side is clearly visible.

Table 6 presents the computed aerodynamic force coefficients including the pressure and viscous contribution for the selected turbulence models compared to the NASA experimental data. Table 6 presents the experimental results for $+10^{\circ}$ and $-10^{\circ}$ yaw angle. The drag and lift coefficients are within the experimental uncertainly of \pm 0.01 , however, the side force coefficient shows much larger difference of 0.128 which quite larger than the suggest value of 0.01 . The computed results were compared to the averaged values of the +10 and -10 yaw angles.

Again the Mentor SST (BSL) model produced the closest drag coefficient prediction $3.9 \%$ to the experimental data. The Wilcox $\mathrm{k}-\mathrm{w}$ was next with $5.4 \%$ followed by the SA model at $17.5 \%$. The SST (BSL) prediction on the coarse mesh was $5.3 \%$ which is similar to $\mathrm{k}-\mathrm{w}$ prediction on the medium mesh. All turbulence models were significantly off about $200 \%-500 \%$ in predicting the lift coefficient compared to the experimental data and even the sign of the lift coefficient were not captured correctly. However, all turbulence models reasonably predicted the side force coefficient within $4.6 \%-5.2 \%$ of the experimental data. The SST (BSL) coarse mesh results generated $5.6 \%$ difference in the side force. Overall, drag coefficient was predicted reasonably except for the SA model, the lift coefficient was no captured at all, and the side force coefficient was adequately captured by all the turbulence models.

To investigate the influence of order of discretiztion of the advective part of the turbulence equations on its predictive capability, two simulations were performed using the SST (BSL) model on coarse and medium mesh in which the advective part of the turbulence model was computed $2^{\text {nd }}$-order accurate. Table 8 presents the computed force coefficients for these two runs. For the drag and lift coefficients the $2^{\text {nd }}$-order accuracy were not helpful and the results slightly moved away from the experiment; however, for the side force coefficient there was a minor improvement. For steady RANS calculations the influence of the $2^{\text {nd }}$-order treatment of the turbulence equation is minor; however, this does not be true for the unsteady RANS flow simulations. 


\begin{tabular}{|c|c|c|c|c|c|c|c|}
\hline Medium grid, SST & & CD & Error & $\mathrm{CL}$ & Error & cs & Error \\
\hline & Pressure & 0.4804 & & -0.0347 & & 1.1458 & \\
\hline & Viscous & 0.0821 & & 0.0027 & & -0.0082 & \\
\hline & Total & 0.5626 & $3.9 \%$ & -0.0320 & $-223 \%$ & 1.1376 & $-5.2 \%$ \\
\hline \multirow[t]{4}{*}{ Medium grid, KW } & & CD & & CL & & cs & \\
\hline & Pressure & 0.4866 & & -0.0263 & & 1.1516 & \\
\hline & Viscous & 0.0842 & & 0.0027 & & -0.0084 & \\
\hline & Total & 0.5708 & $5.4 \%$ & -0.0236 & $-190 \%$ & 1.1431 & $-4.7 \%$ \\
\hline \multirow[t]{4}{*}{ Medium grid, SA } & & CD & & CL & & CS & \\
\hline & Pressure & 0.5641 & & -0.1192 & & 1.1529 & \\
\hline & Viscous & 0.0721 & & 0.0030 & & -0.0078 & \\
\hline & Total & 0.6361 & $17.5 \%$ & -0.1162 & $-547 \%$ & 1.1451 & $-4.6 \%$ \\
\hline \multirow[t]{4}{*}{ Coarse grid, SST } & & CD & & CL & & CS & \\
\hline & Pressure & 0.4866 & & -0.0281 & & 1.1414 & \\
\hline & Viscous & 0.0835 & & 0.0029 & & -0.0084 & \\
\hline & Total & 0.5701 & $5.3 \%$ & -0.0252 & $-197 \%$ & 1.1329 & $-5.6 \%$ \\
\hline \multirow[t]{3}{*}{$\begin{array}{c}\text { NASA experiment } \\
\pm 0.01 \text { error }\end{array}$} & & CD & & CL & & CS & \\
\hline & Point 5 & 0.54 & $\pm 1.8 \%$ & 0.022 & $\pm 38.4 \%$ & 1.264 & $\pm 0.8 \%$ \\
\hline & Point 15 & 0.543 & & 0.03 & & -1.136 & \\
\hline Averaged & & 0.5415 & & 0.026 & & 1.2 & \\
\hline
\end{tabular}

Table 7: Computed aerodynamic forces for the GTS model at $10^{\circ}$ yaw including the NASA experimental data

\begin{tabular}{|l|l|c|c|c|c|c|c|}
\hline Medium grid, SST, $2^{\text {nd }}$-order & & CD & Error & CL & Error & CS & Error \\
\hline & Pressure & 0.4858 & & -0.0553 & & 1.1547 & \\
\hline & Viscous & 0.0835 & & 0.0027 & & -0.0080 & \\
\hline & Total & 0.5701 & $4.4 \%$ & -0.0526 & $-302 \%$ & 1.1466 & $-4.4 \%$ \\
\hline Coarse grid, SST, 2 ${ }^{\text {nd }}$-order & & CD & & CL & & CS & \\
\hline
\end{tabular}




\begin{tabular}{|c|l|l|l|l|l|l|l|}
\hline & Pressure & 0.4937 & & -0.0495 & & 1.1449 & \\
\hline & Viscous & 0.0795 & & 0.0029 & & -0.0083 & \\
\hline & Total & 0.5745 & $6.1 \%$ & -0.0466 & $-279 \%$ & 1.1366 & $-5.3 \%$ \\
\hline $\begin{array}{c}\text { NASA experiment } \\
\pm 0.01 \text { error }\end{array}$ & & CD & & CL & & CS & \\
\hline & Point 5 & 0.54 & $\pm 1.8 \%$ & 0.022 & $\pm 38.4 \%$ & 1.264 & $\pm 0.8 \%$ \\
\hline & Point 15 & 0.543 & & 0.03 & & -1.136 & \\
\hline & & 0.5415 & & 0.026 & & 1.2 & \\
\hline Averaged & & & & &
\end{tabular}

Table 8: Computed aerodynamic force coefficients for the GTS model at $10^{\circ}$ yaw including the NASA experimental data, $2^{\text {nd }}$-order treatment of the advective part of the SST turbulence model.

Let's examine the pressure field on the body of GTS at this yaw angle by comparing it to the experiment data. Figure 40-46 presents the comparison of the computed pressure coefficients on the symmetry plane $(y / w=0)$, for all turbulence models, to the pressure tap data of the NASA experiment. The uncertainty for the computed $\mathrm{C}_{\mathrm{p}}$ from the experiment is about 0.004 [4]. This uncertainty is not shown as the error bar because it size is similar to the symbols size used in these figures. Figure 40 compares the computed $\mathrm{C}_{\mathrm{p}}$ in front of GTS (stagnation area) to the pressure tap data and the agreement is reasonable. Figure 41 compares the pressure coefficient distribution on top of GTS to the experimental data. The agreement is reasonable except for the area close to the base of the trailer where SST and k-w models depart significantly from the data. However, SA has a right trend but does not fully capture the data. This behavior was observed not with the same severity at the $0^{\circ}$ yaw. There is something happing on the top of the trailer close to the base in the experimental data that the computational models are not predicting. As was stated earlier, the correct simulation of this area is crucial in capturing the trailer wake flow structure. Figure 42 shows the comparison of $C_{p}$ on the bottom of GTS to the experimental data. The pressure recovery under the tractor does not match the experiment but the computed result has the right trend. Under the trailer the computed result does not have the right trend compared to the experimental data. However, the agreement improves towards the base of the trailer.

Figure 43 presents the comparison of $\mathrm{Cp}$ on the left side (looking up) of GTS compared to the experimental data. There is a slight disagreement around the stagnation area which it improves toward the base of the trailer. This suggests possible small variation in the yaw angle. Figure 44 presents the comparison of $\mathrm{Cp}$ to the experiment on the lee-ward side of the GTS. The agreement is reasonable on the trailer; however, there is a minor disagreement the tractor side close to the separation bubble. Figure 45 shows the $\mathrm{Cp}$ distribution on the base of the trailer compared to the experimental data. There is a significant disagreement between the computed result in both trend and the magnitude compared to the experimental data. 
Figure 46 shows the pressure distribution on the base of the trailer from the symmetry plane toward the edge of the trailer. The disagreement between the computed and the experiment is quite visible covering the half of the trailer surface.

The $2^{\text {nd }}$-order treatment of the turbulence equations did not altered the pressured field on the body of GTS except for the base as shown in Fig 47. this minor variations are responsible for the change in drag prediction as shown in table 8.

Overall, all turbulence models adequately captured the pressure field on the body of GTS except for the top and the base of the trailer.

To investigate the mesh resolution influence on the pressure field, similar to the $0^{\circ}$ yaw, a coarse mesh simulation is performed using the SST (BSL) turbulence model. Figures 47-49 present grid convergence study for SST baseline turbulence model at the $10^{\circ}$ yaw. Both coarse and medium grids produced a similar solution around the GTS except for the base of the trailer where there is a difference in the solution. This suggested the pressure field around the GTS has adequately being resolved.

To investigate the trailer wake flow structure, let's compare computed solutions to the time-averaged PIV data. Figure 51 presents the PIV data using particle traces for the horizontal laser light sheet located at the trailer mid-height. This figure suggests the presence of three vortices in the wake. The SST and SA models (Figs. 52 and 54) both capture two vortices but show no possibility of a third. The KW model shown in Fig 53 does not display either of the vortices on the leeward side of the wake; however, the unusual trace pattern suggests that some of the vortex effects are captured. Among the models that captured windward vortices, SA over-predicts the longitudinal and spanwise distance from the rear edge by $25 \%$ and $115 \%$. KW shows the same vortex to be closer to the model centerline over-predicting the same values by roughly $20 \%$ and $200 \%$. SST does the best job predicting the windward vortex location underestimating its distance from the base of the model by $5 \%$. Unfortunately, like SA and KW, SST is unable to place the vortex in the proper spanwise location, over-predicting the distance to the model edge by $105 \%$. Among the modeled that captured leeward vortices, SA and BSL exhibit opposite behavior. SA over-predicts the longitudinal location by $30 \%$ but is within $1 \%$ of the spanwise location. In contrast, SST under-predicts the longitudinal location by less than $5 \%$, but the spanwise distance is $30 \%$ smaller than suggested by PIV. The vertical laser light sheet plane located at $\mathrm{x} / \mathrm{w}=$ ???, shown in Fig. 55, 14.5" from the rear of the yawed model highlights a dominant vortex present in the experimental data. Both KW (Fig. 57) and SA (Fig 58) display similar impinging shear layers, but only SST, shown in Fig. 56, predicts a large roll-up near the location shown in the PIV data.

Given the above comparison the computed wake flow structure is significantly different from the PIV data; however it is interesting to note that the integrated pressure field on the base of the trailer produces a reasonable drag force. 
To further examine the trailer wake flow structure, tunnel-aligned velocity components were extracted along the GTS model centerline for all computed solutions, as shown in Figure 59, for comparison to the PIV data set. For the $10^{\circ}$ yaw velocities were normalized by non-dimensional free-stream velocity of .281. Similar to the $0^{\circ}$ yaw results, all turbulence models consistently over-predict the velocity magnitude in the recirculation region. The observed discrepancy in the centerline velocity extracted from different laser sheet of the PIV data should be investigated.

At this yaw angle multiple vortex structures are present in the vicinity of the GTS body as shown in Fig 39. The position of the vortex that rolls-up on top of the trailer is compared quantitatively to the surface shear stress vector data (Fig. 60) obtained from the oil film interferometry. Figures 61-63 presents the particle traces confined to the top of the GTS for all turbulence model calculations. Two lines are drawn over these images representing the separation and reattachment lines obtained from the experimental data.! Noted that there is an uncertainty associated with the position of these lines, since a relatively coarse surface shear stress vector data was used to extract them. All turbulence models clearly predicted the presence of the main vortex and there were indication of the existence of the second vortex. Among the turbulence models SA predicted the closest separation and the reattachment lines to the experimental data with SST and KW being slightly off for the main vortex and they missed the position of the second vortex.

On the leeward side of the GTS there exists a separation bubble as shown in Fig. 64 . To investigate the turbulence model predictions on this side of GTS, computational results are shown in Figs. 65-67 as particle traces confined to this surface. The SST and $\mathrm{KW}$ models show analogous separation and reattachment patterns suggesting a similar size separation bubble; however, the SA model predicts significantly different solution and the bubble size. 


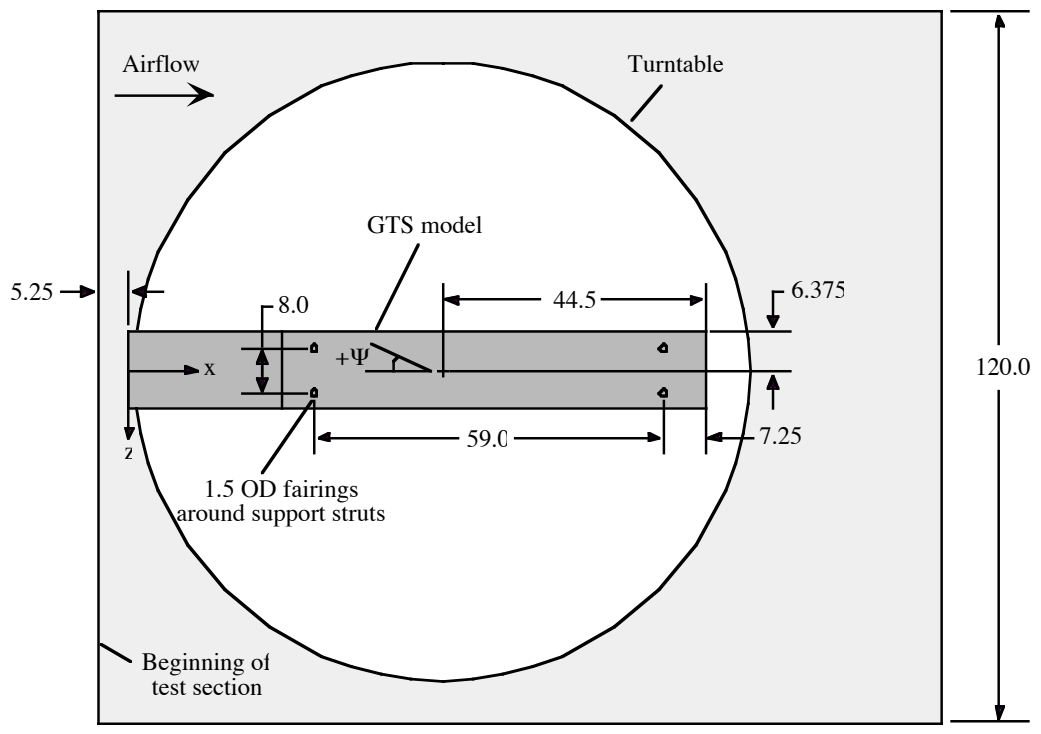

Figure 1: GTS model installation top view ${ }^{5}$
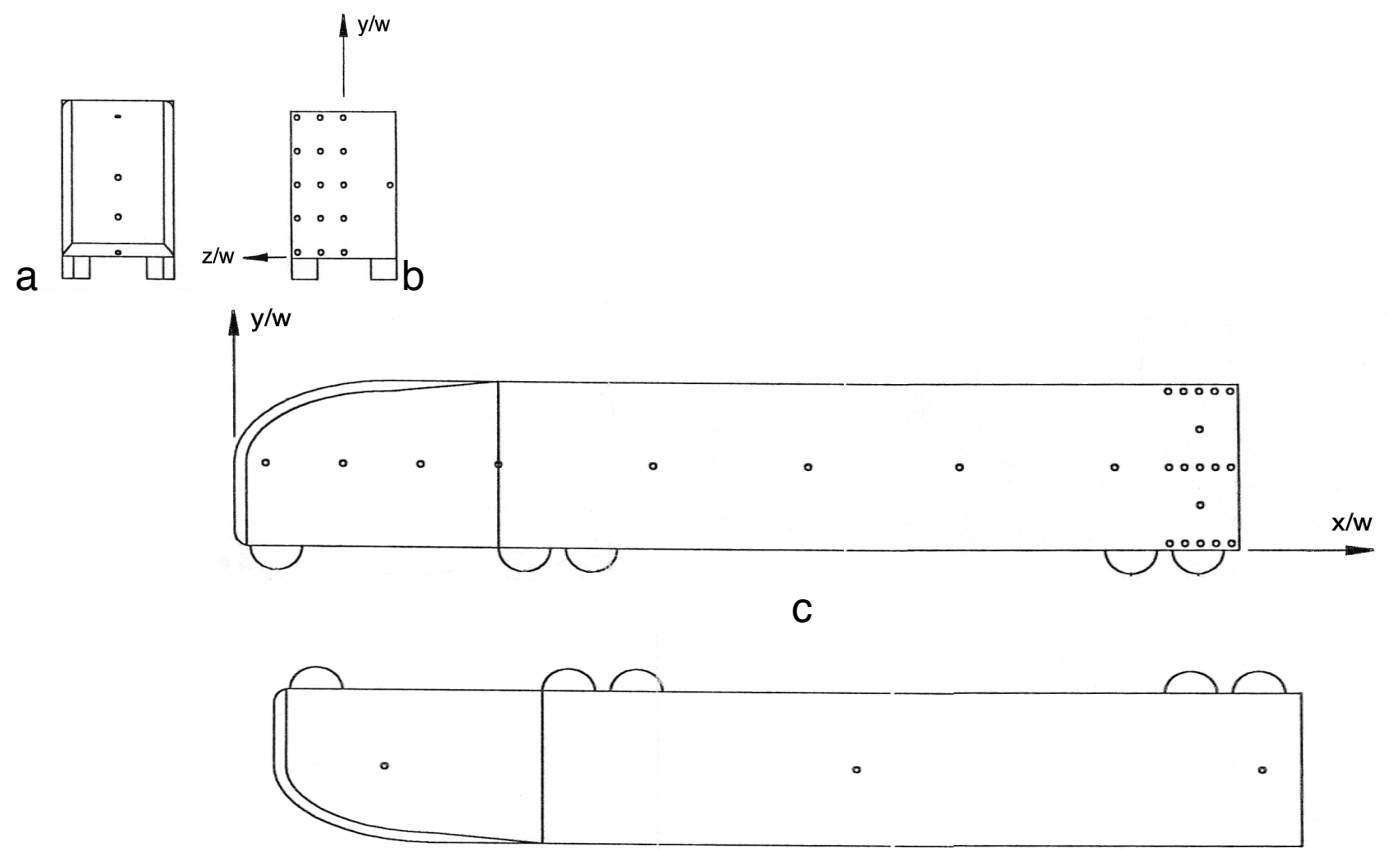

d

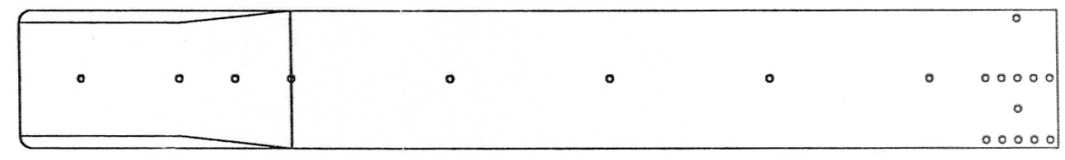


e

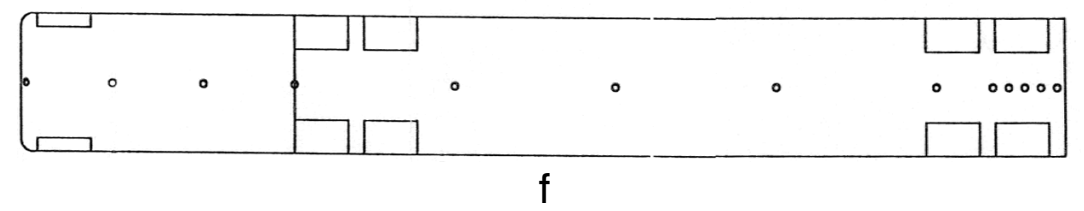

Figure 2: Pressure tap locations on the GTS model, a) front, b) base, c) port side, d) starboard side, e) top, and f) bottom.

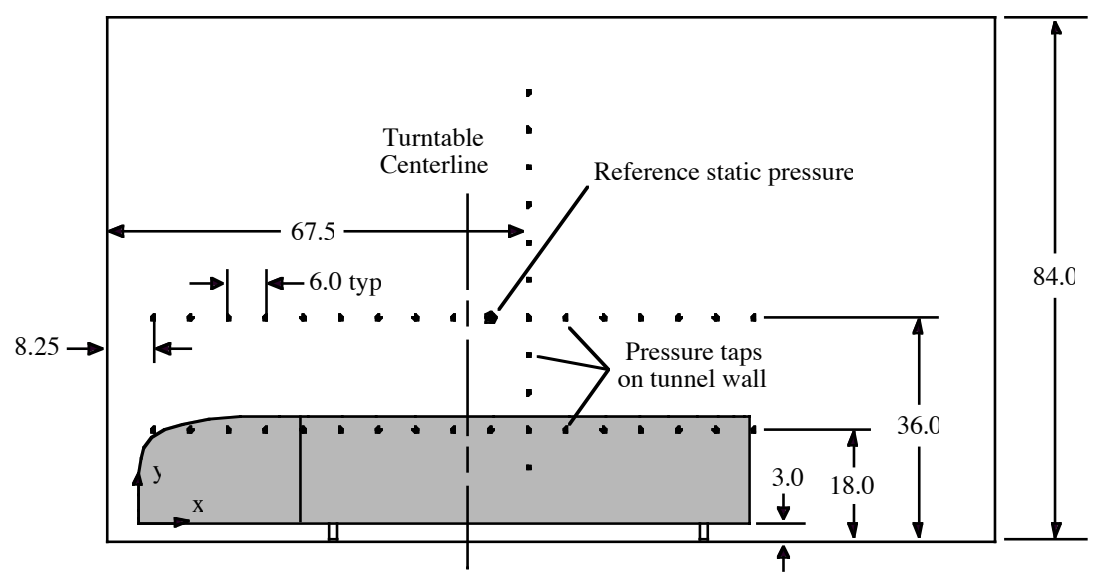

Figure 3: GTS model installation side view (dimensions in inches). ${ }^{5}$ 


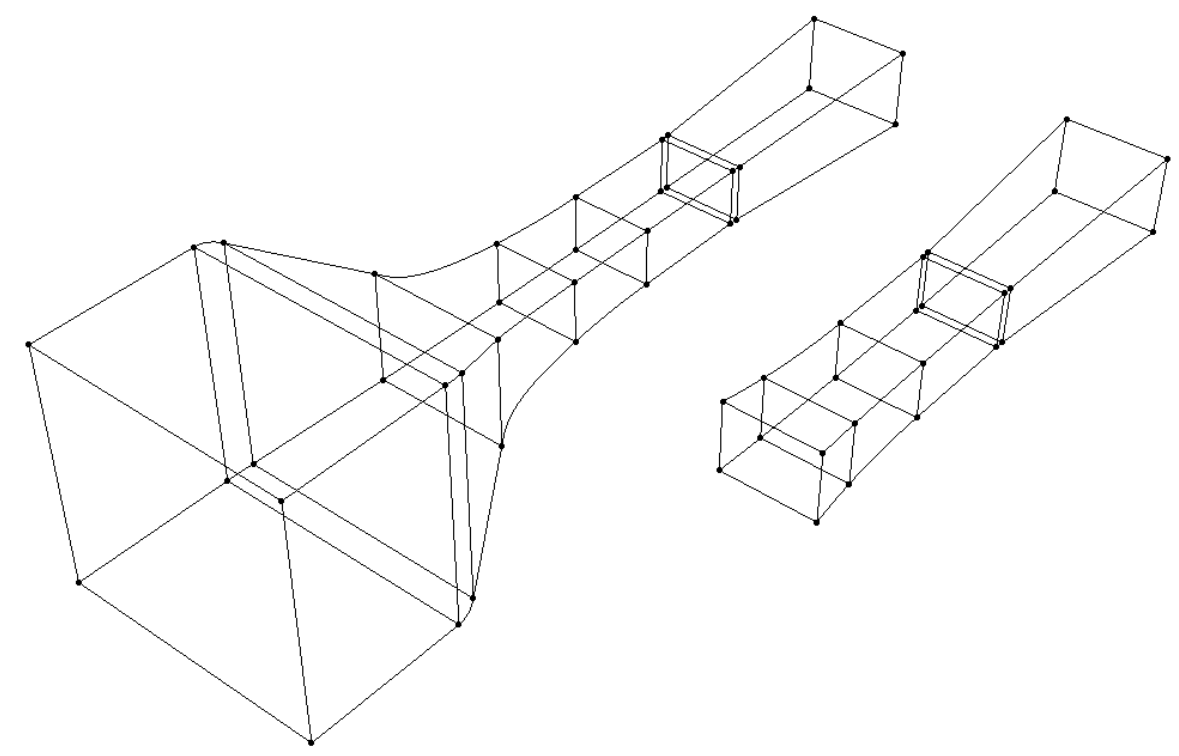

Figure 4. a) Model of NASA Ames wind tunnel including the settling chamber, and b) truncated model._LABEL A) AND B)!!!!!!!!!!!!!!!

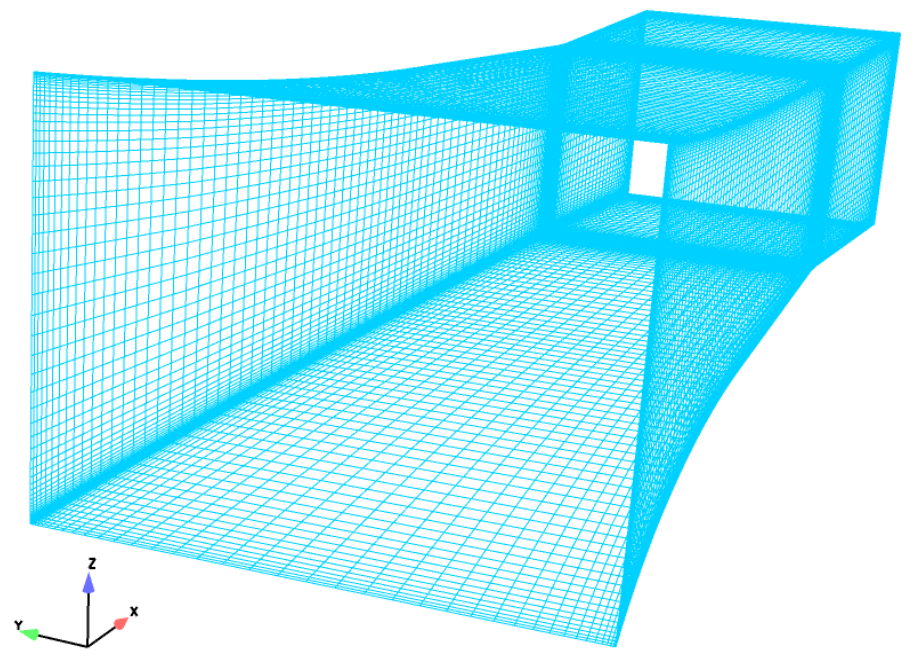

Figure 5. Empty tunnel coarse mesh, 466,240 elements. 


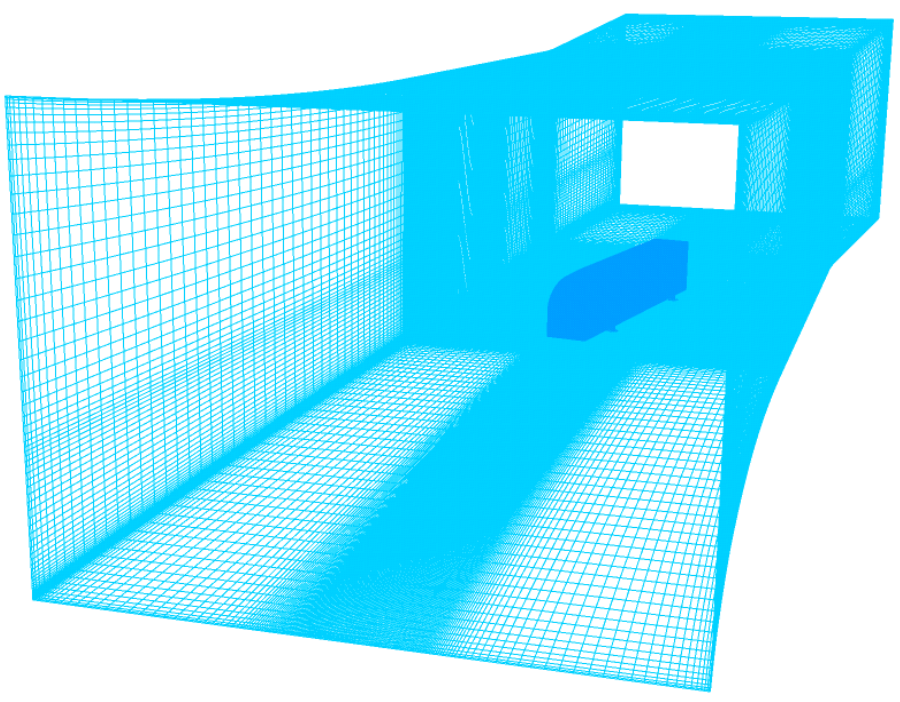

Figure 6. GTS in NASA 7'x10' tunnel, coarse mesh, $10^{\circ}$ yaw.

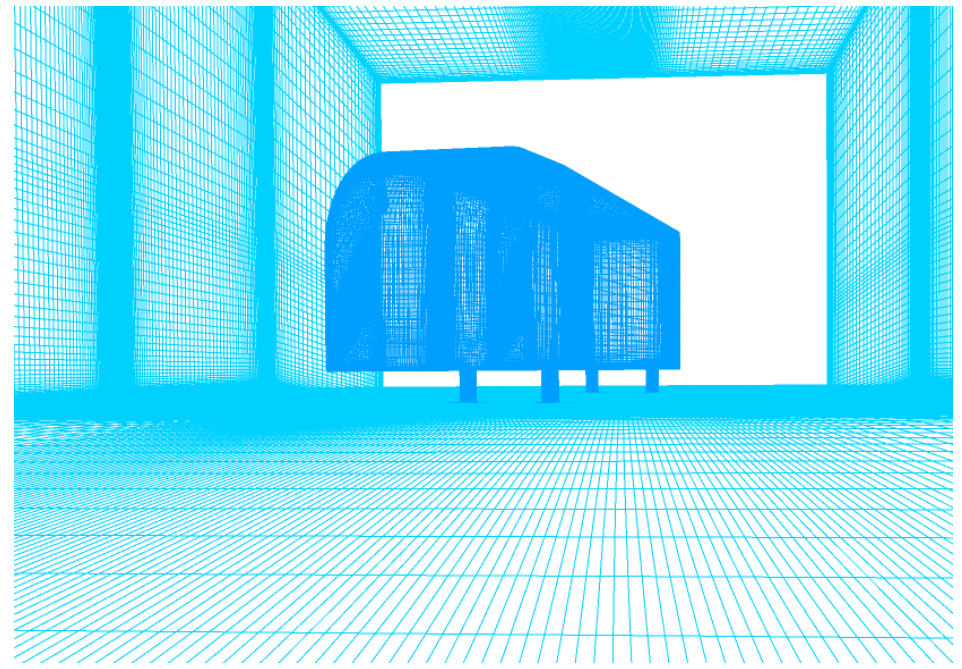

Figure 7. GTS in tunnel mesh, coarse grid, $10^{\circ}$ yaw. 


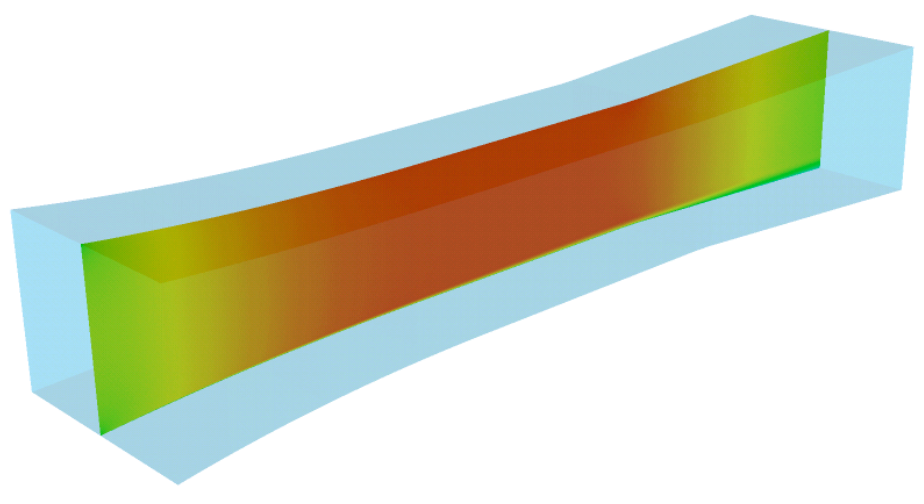

Figure 8. Empty tunnel simulation, Mach contours, symmetry plane.

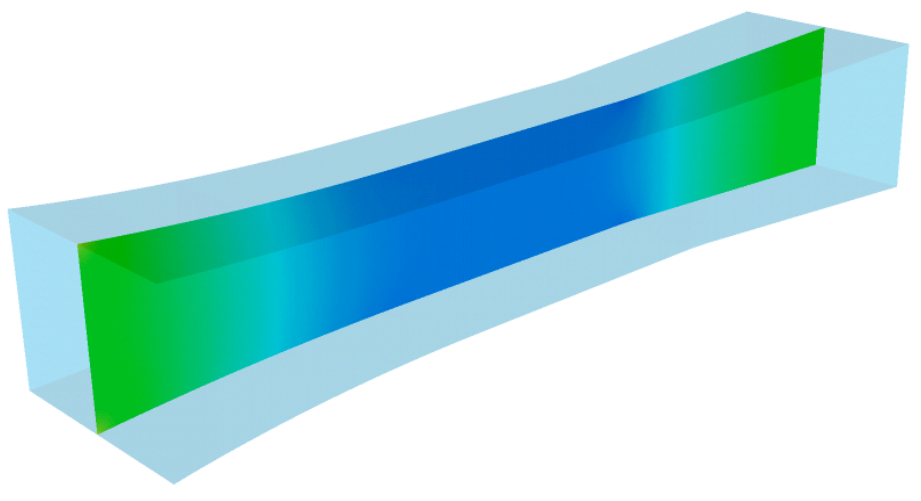

Figure 9. Empty tunnel simulation, pressure contours, symmetry plane. 


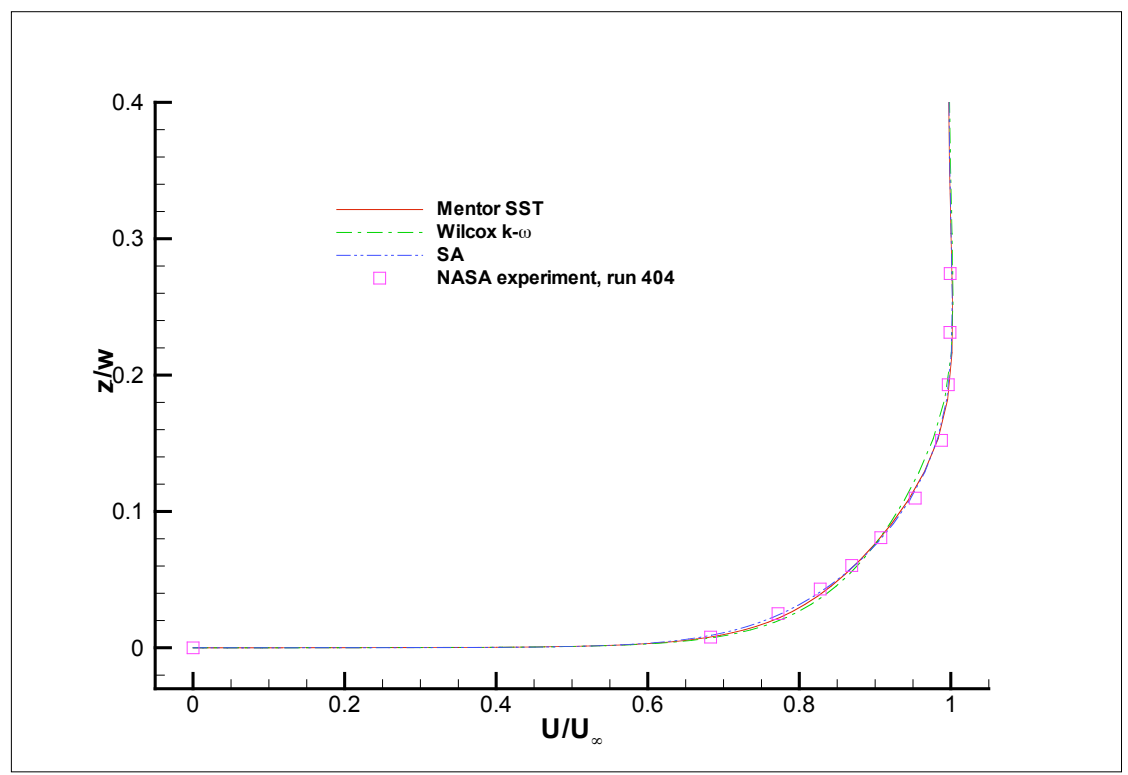

Figure 10. Empty tunnel simulations, comparison of the computed boundary layer profiles to the NASA Ames experimental data.

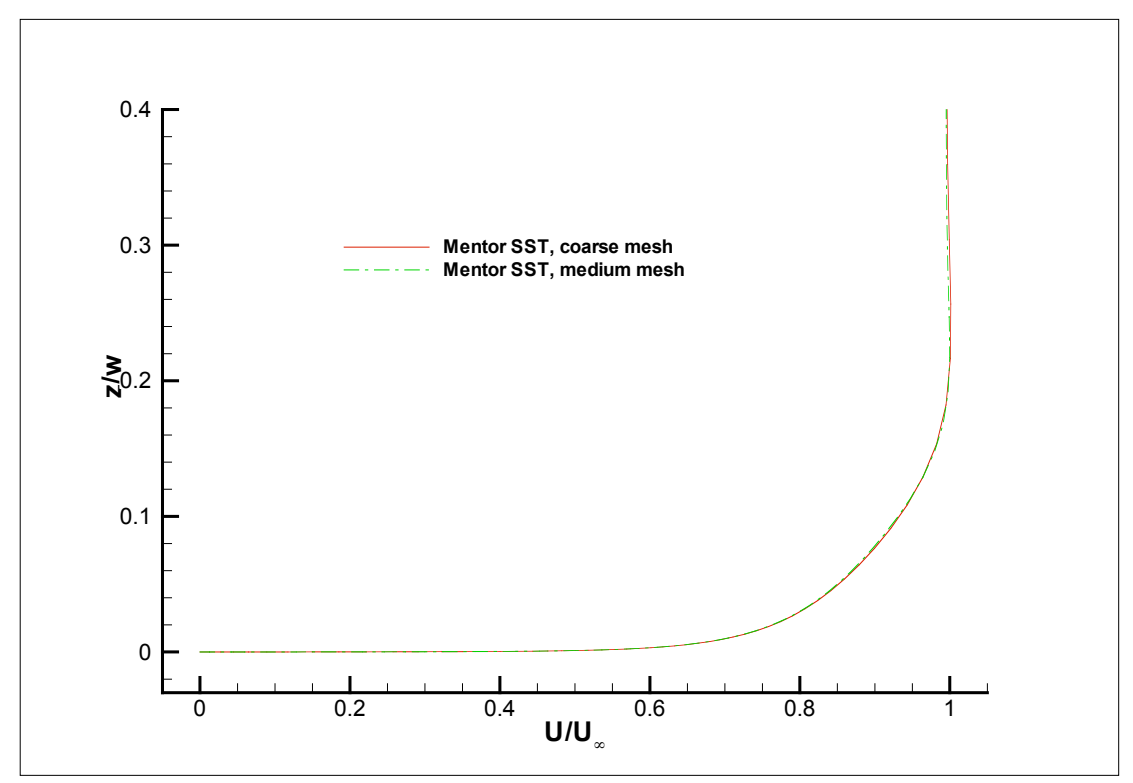

Figure 11. Empty tunnel simulations, grid convergence study. 


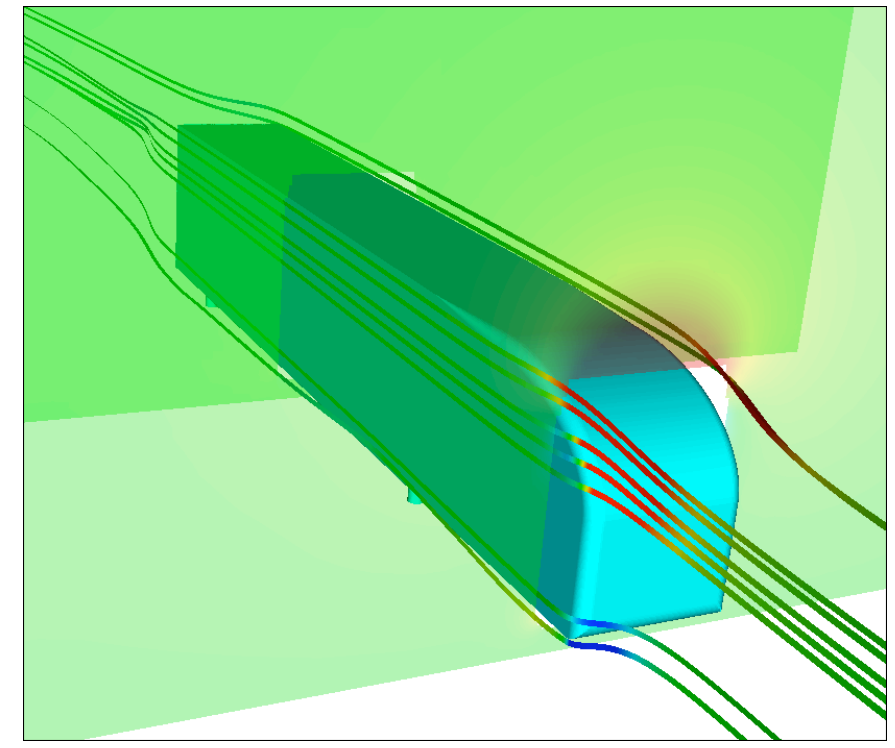

Figure 12: GTS model at $0^{\circ}$ yaw, particle traces colored by velocity magnitude.

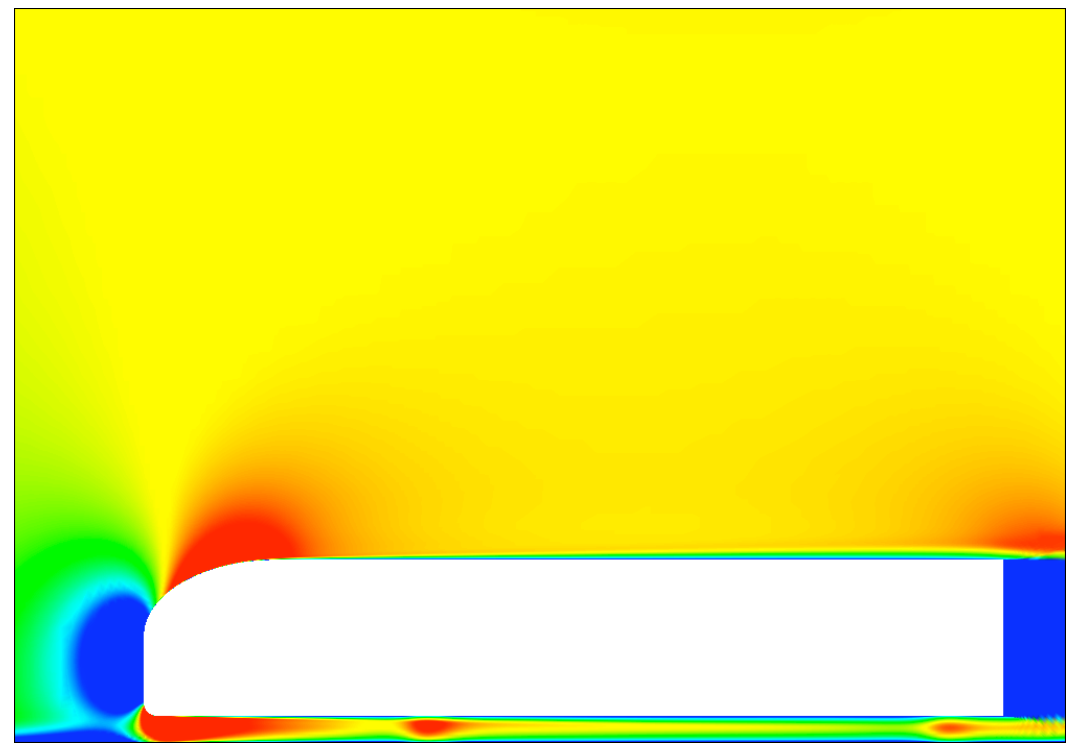

Figure 13: Velocity magnitude contour, symmetry plane $(y / w=0), 0^{\circ}$ yaw. 


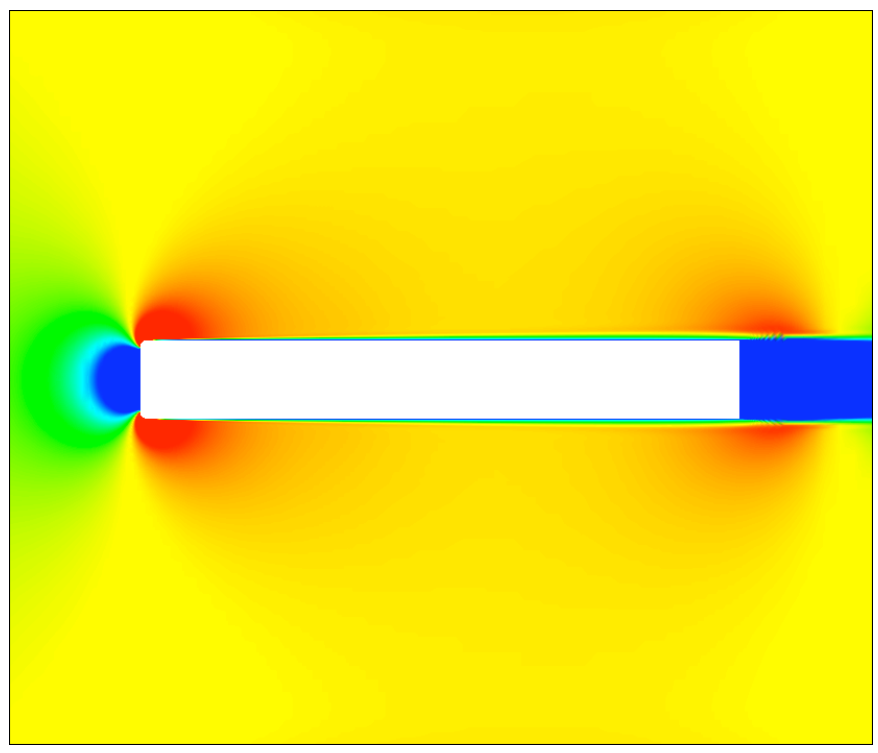

Figure 14: Velocity magnitude contour, horizontal plane $(\mathrm{z} / \mathrm{w}=?$ ? $), 0^{\circ}$ yaw.

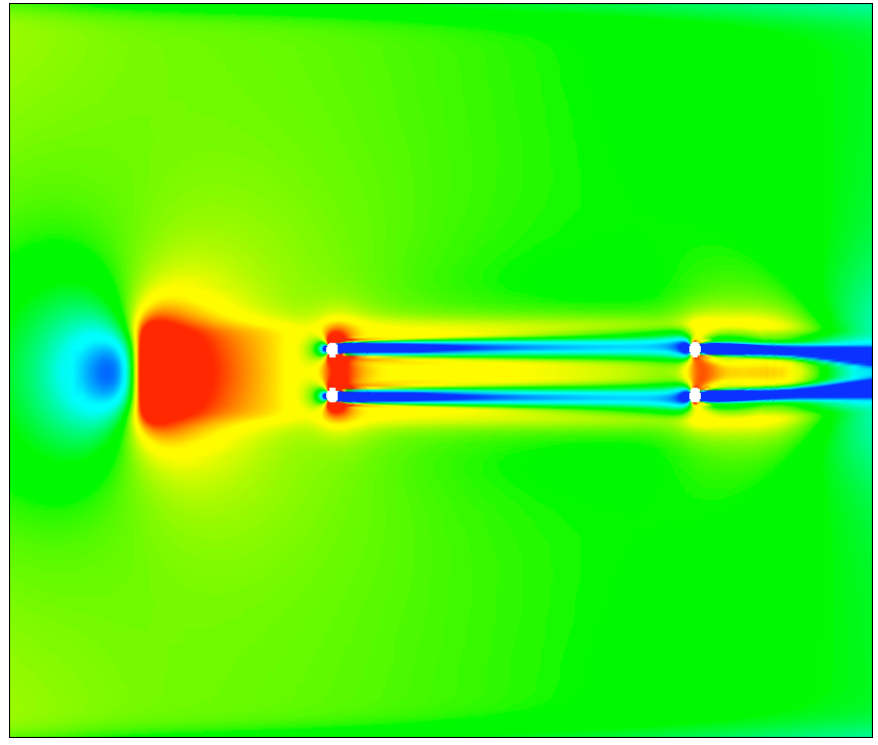

Figure 15: Velocity magnitude contour, horizontal plane $(\mathrm{z} / \mathrm{w}=?$ ? $), 0^{\circ}$ yaw. 


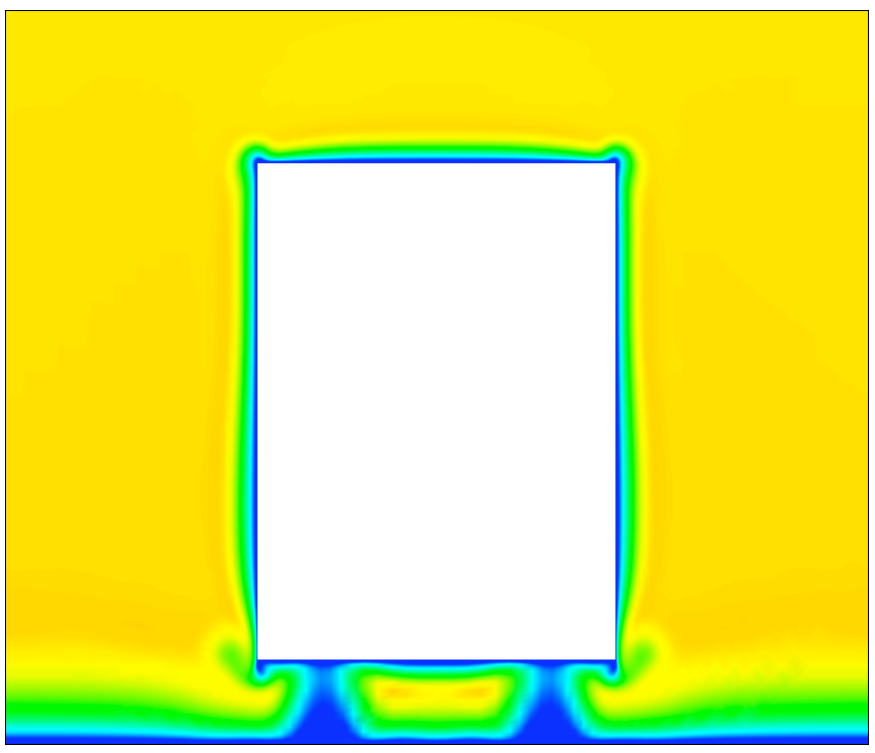

Figure 15: Velocity magnitude contour, vertical plane ( $\mathrm{x} / \mathrm{w}=$ ???), $0^{\circ}$ yaw.

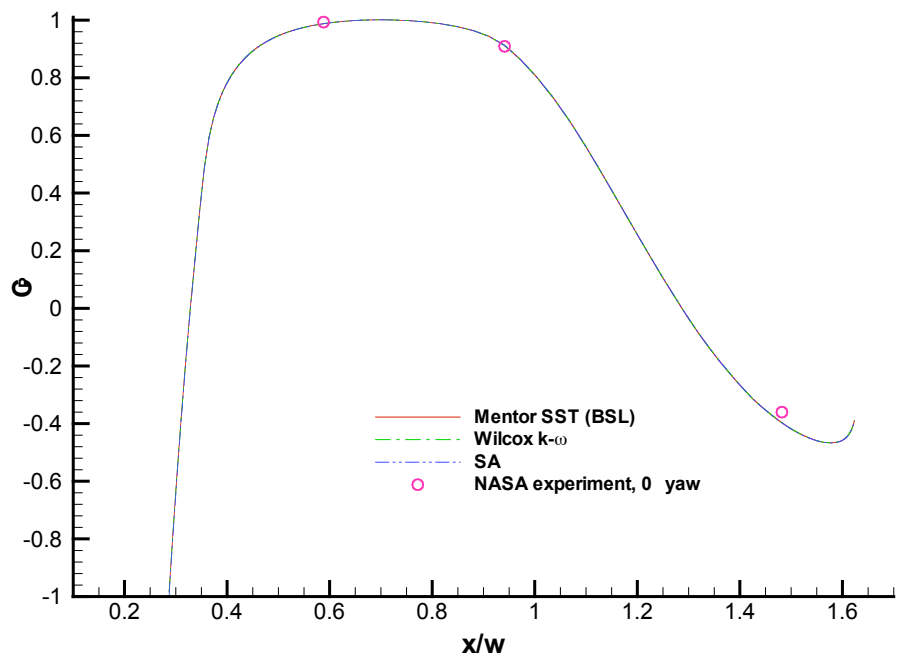

Figure 16: Comparison of the computed $C_{p}$ to the NASA experimental data, front of GTS, $0^{\circ}$ yaw. 


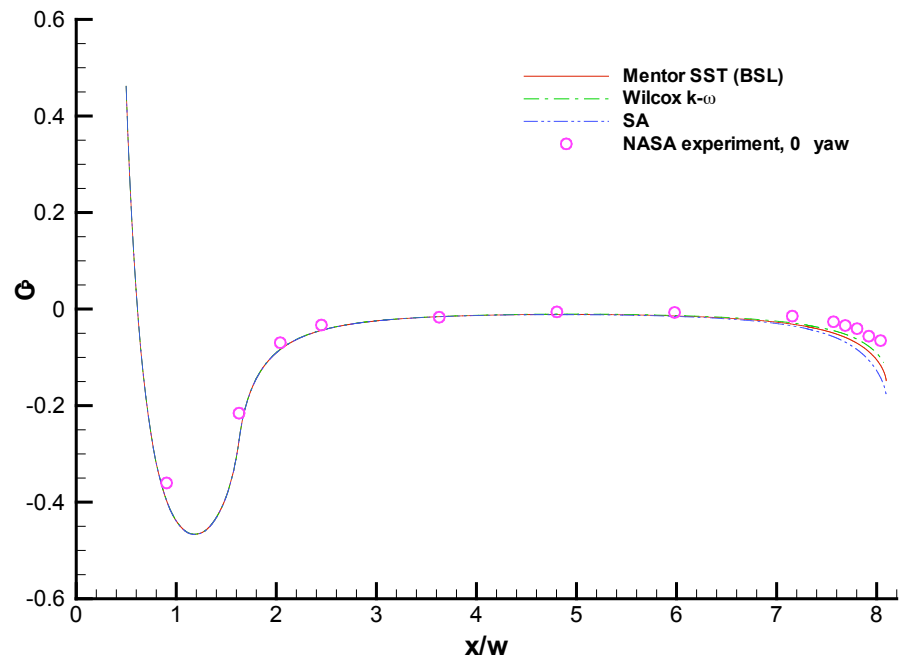

Figure 17: Comparison of the computed $C_{p}$ to the NASA experimental data, top of GTS, $0^{\circ}$ yaw.

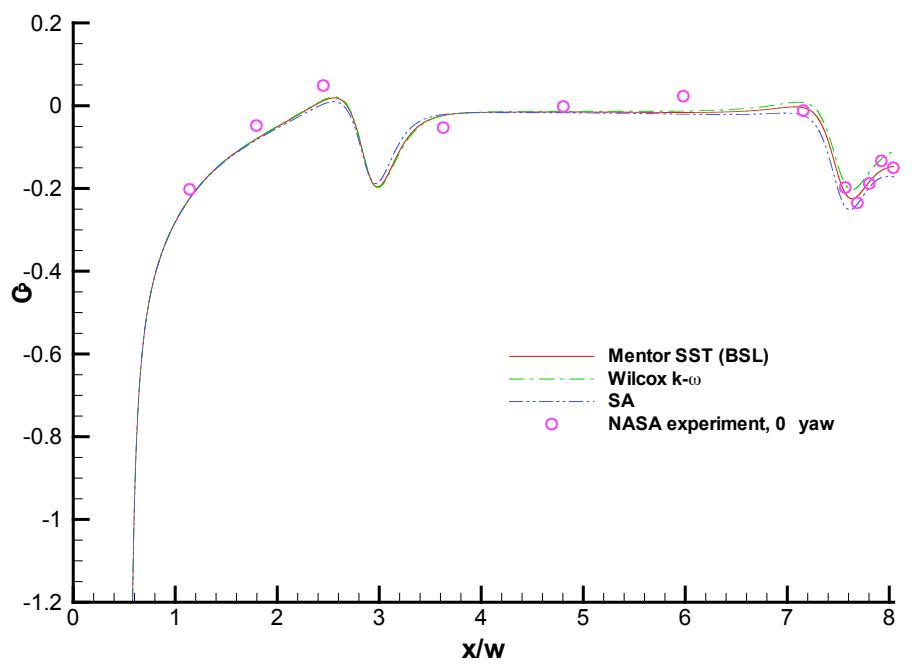

Figure 18: Comparison of the computed $C_{p}$ to the NASA experimental data, under the GTS, $0^{\circ}$ yaw. 


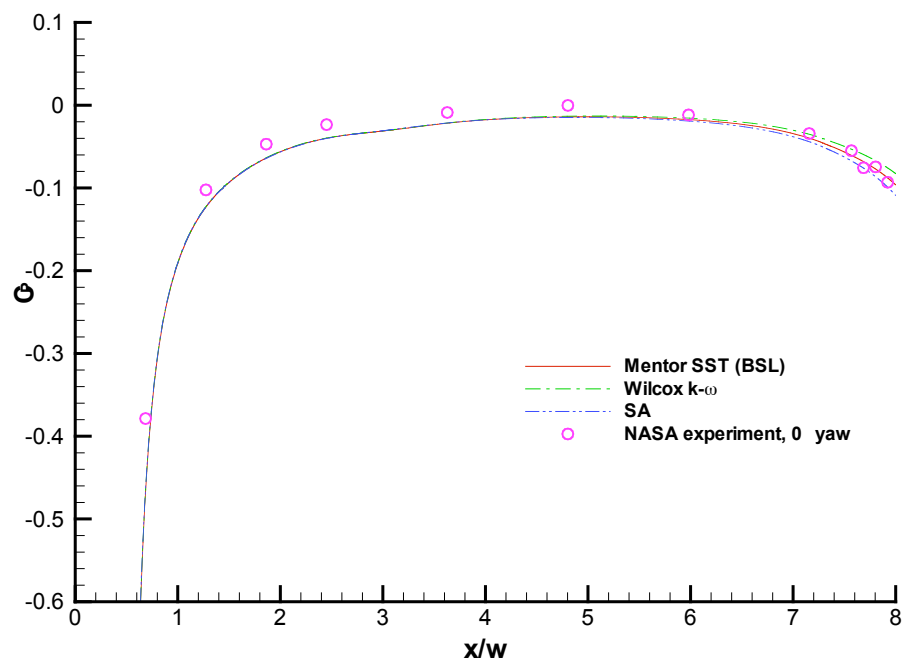

Figure 19: Comparison of the computed $C_{p}$ to the NASA experimental data, side of GTS, $0^{\circ}$ yaw.

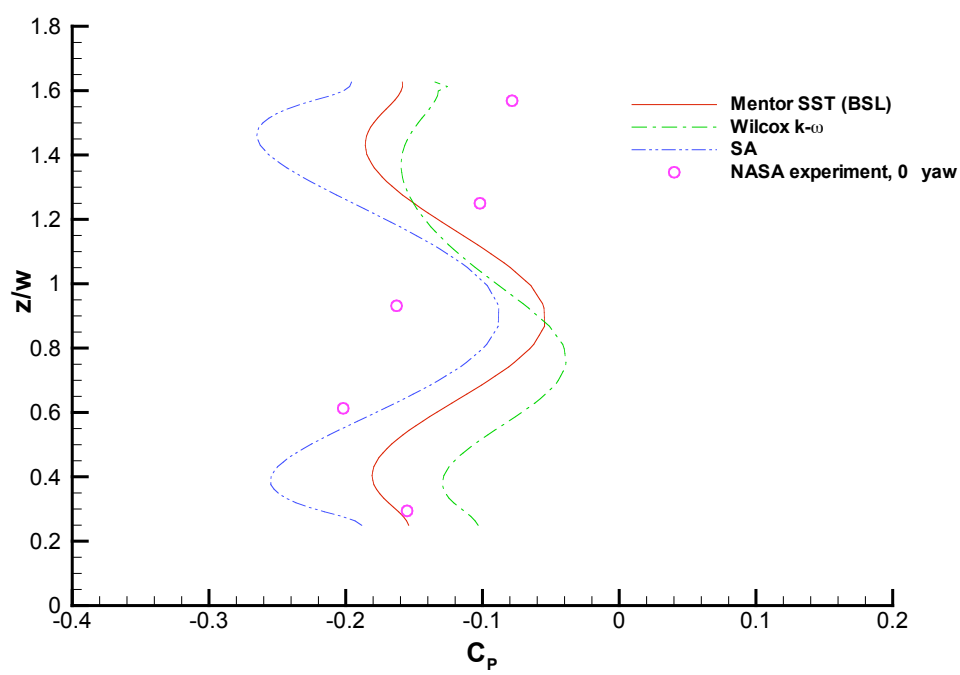

Figure 20: Comparison of the computed $\mathrm{C}_{\mathrm{p}}$ to the NASA experimental data, base of the GTS, $0^{\circ}$ yaw. 


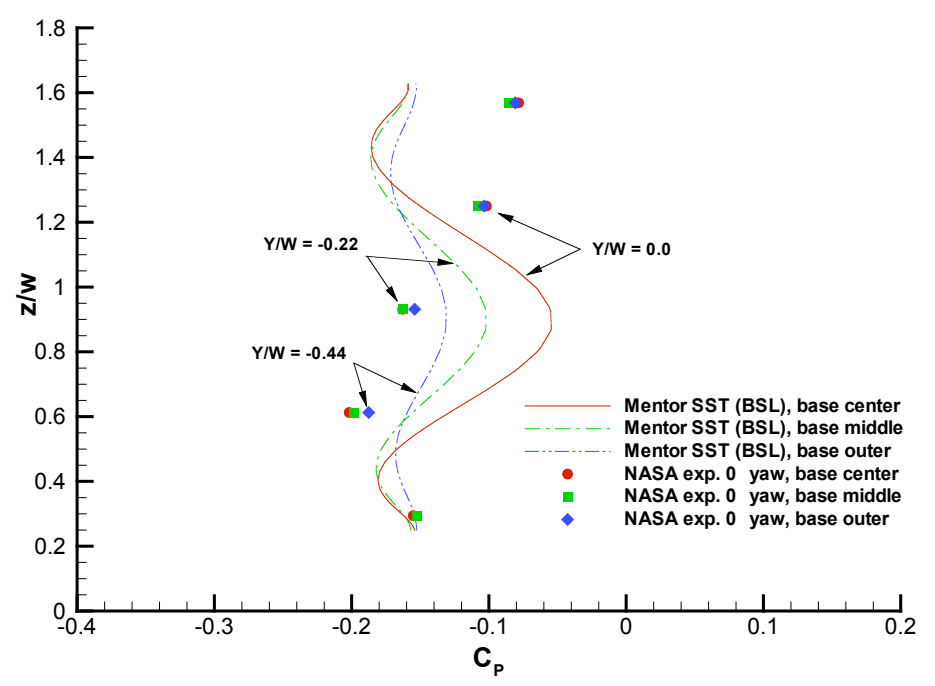

Figure 21: Comparison of the computed $C_{p}$ to the NASA experimental data, base of GTS with all pressure taps, $0^{\circ}$ yaw.

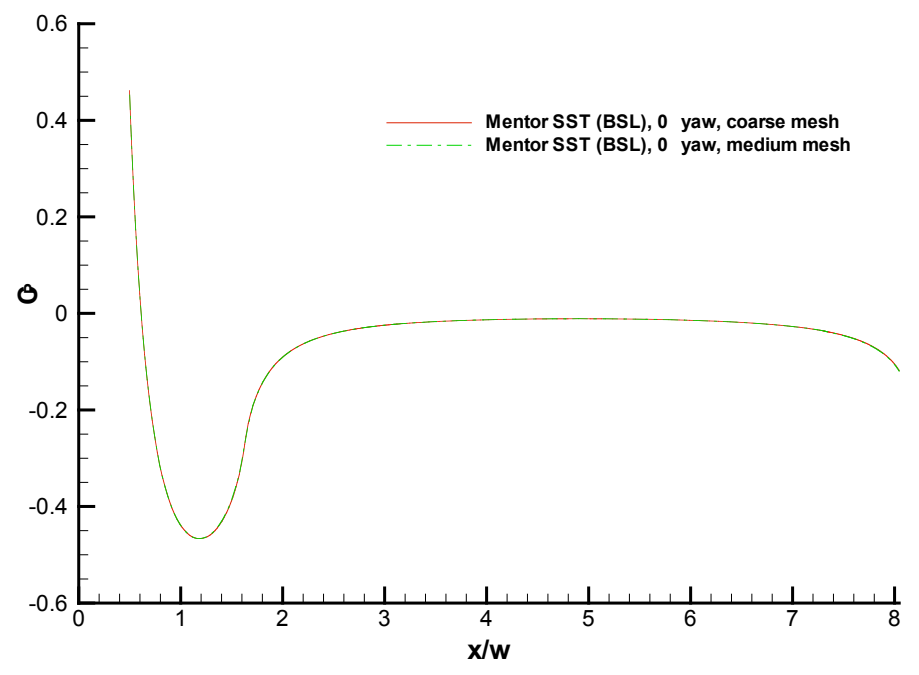

Figure 22: Grid resolution study with Mentor SST turbulence model, top of the GTS, $0^{\circ}$ yaw. 


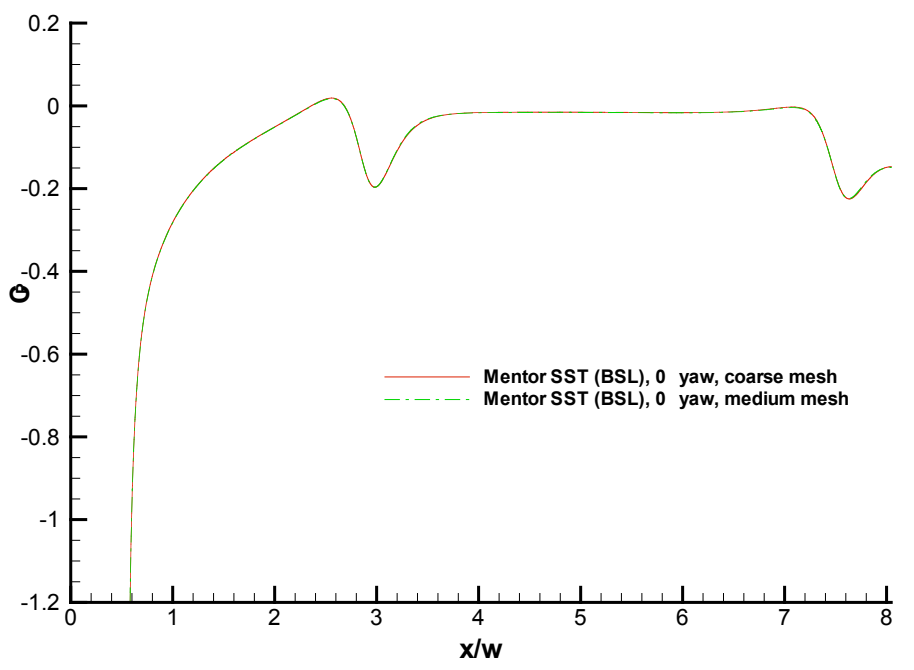

Figure 23: Grid resolution study with Mentor SST turbulence model, under the GTS, $0^{\circ}$ yaw.

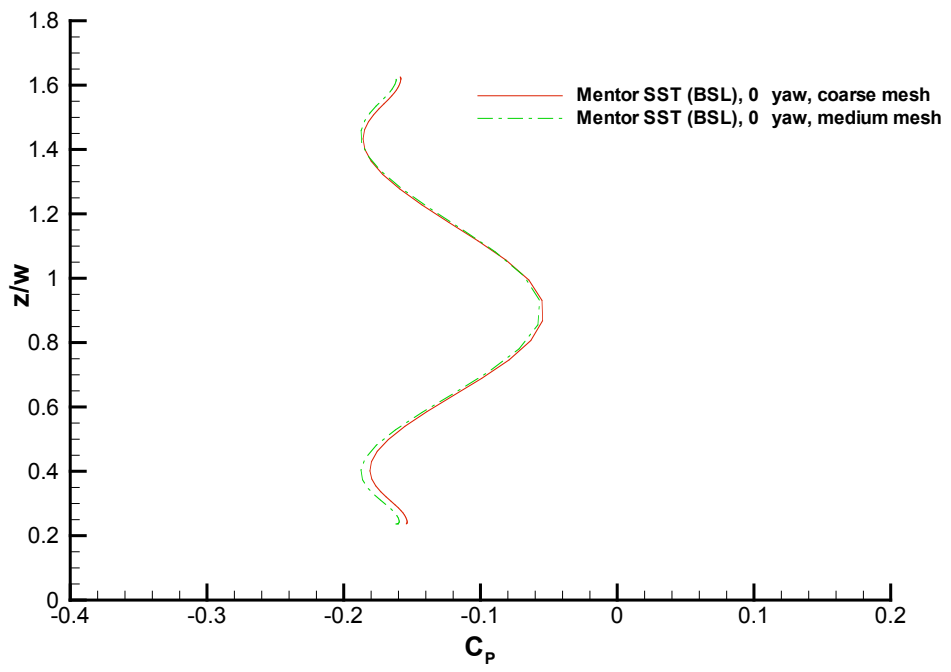

Figure 24: Grid resolution study with Mentor SST (BSL) turbulence model, base of the GTS, $0^{\circ}$ yaw. 


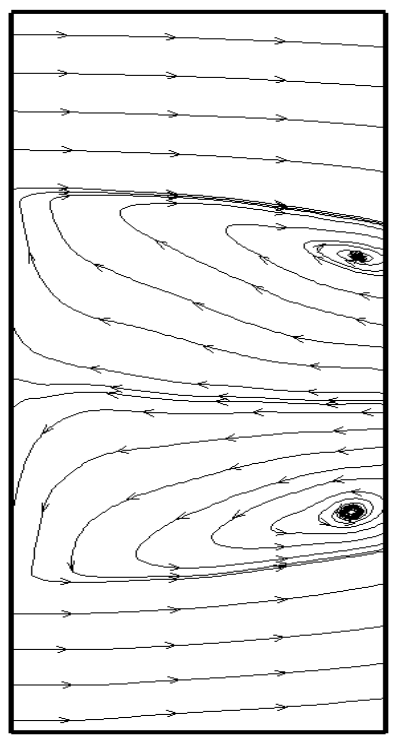

Figure 25: time-averaged PIV data, particle traces, horizontal laser sheet located at trailer mid-height, $0^{\circ}$ yaw.

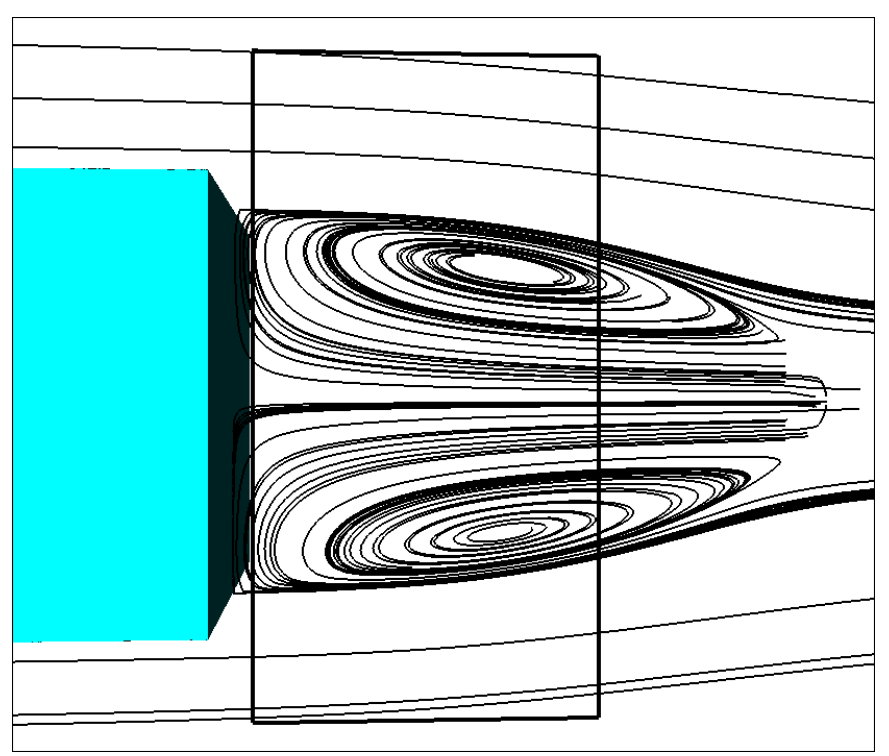

Figure 26: Particle traces, horizontal cut-plane at trailer mid-height, Mentor SST (BSL) solution, $0^{\circ}$ yaw. 


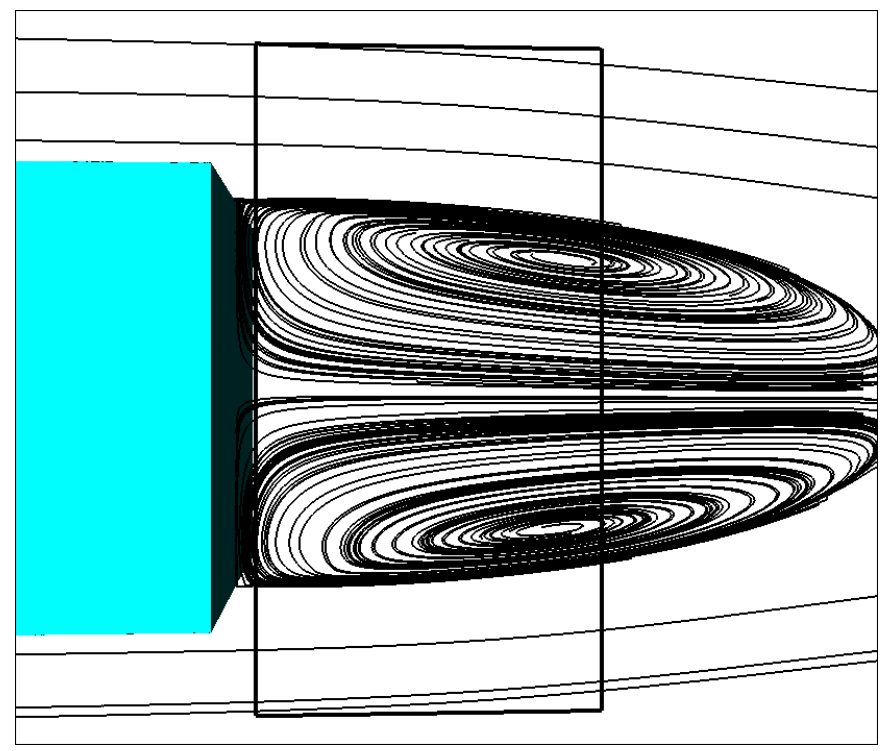

Figure 27: Particle traces, horizontal cut-plane at trailer mid-height, Wilcox k-w solution, $0^{\circ}$ yaw.

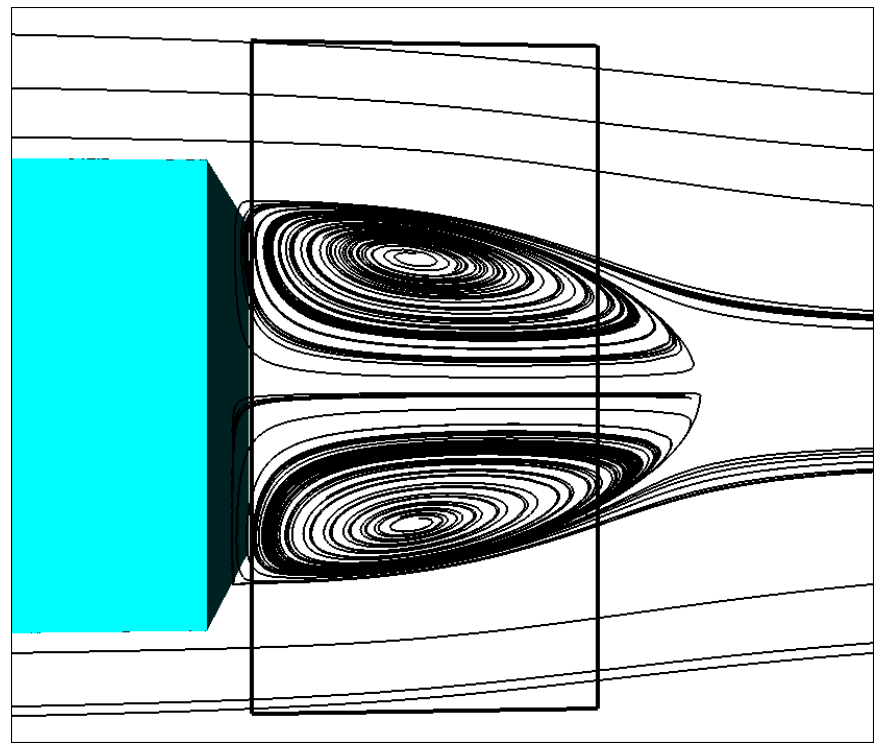

Figure 28: Particle traces, horizontal cut-plane at trailer mid-height, SA solution, $0^{\circ}$ yaw. 


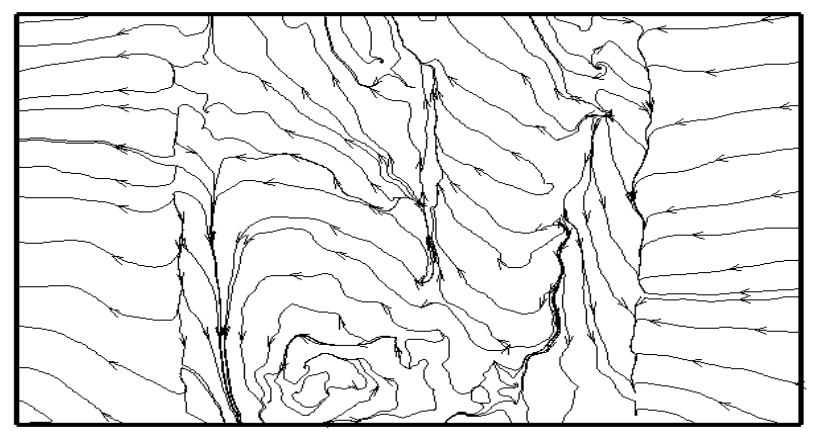

Figure 29: Particle traces time-averaged PIV data, vertical laser light sheet parallel to the base of the trailer located at $x / w=$ ???, $0^{\circ}$ yaw.

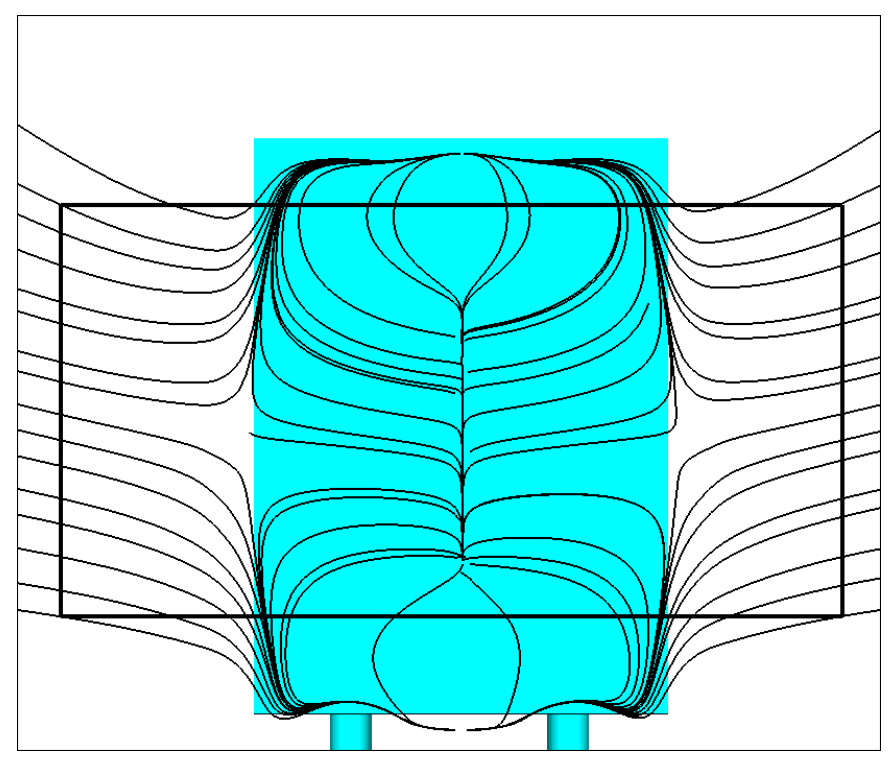

Figure 30: Particle traces SST (BSL) solution, vertical cut-plane parallel to the base of the trailer, $0^{\circ}$ yaw. 


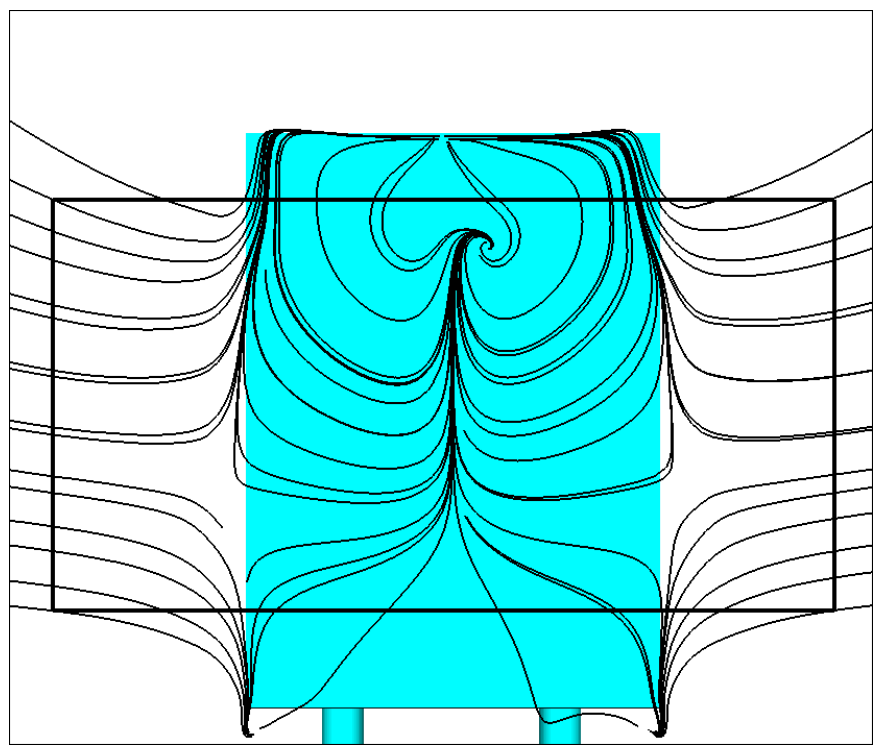

Figure 31: Particle traces Wilcox k-w solution, vertical cut-plane parallel to the base of the trailer, $0^{\circ}$ yaw.

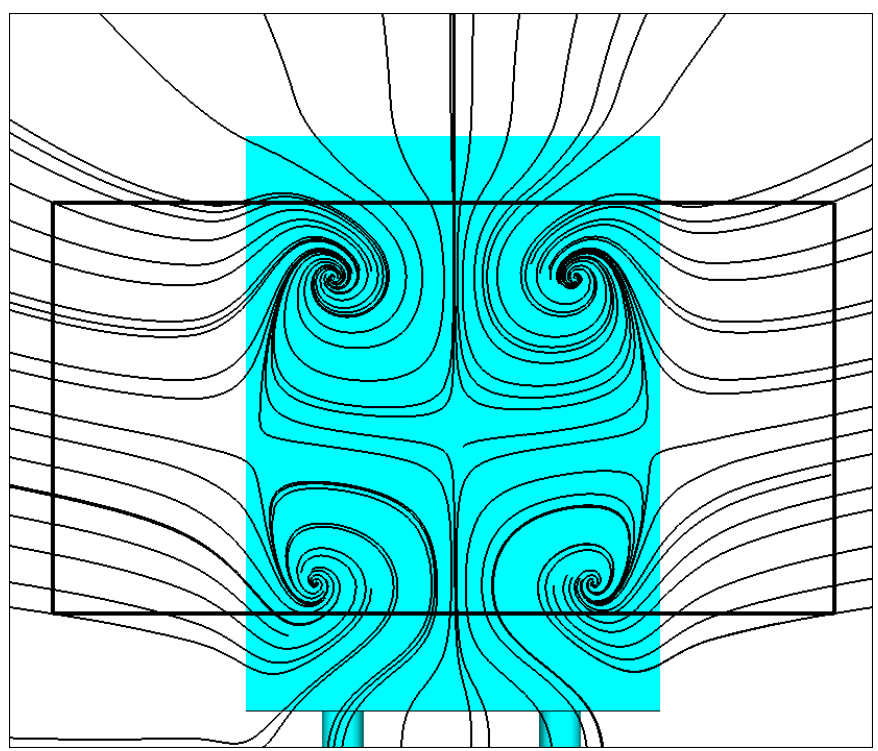

Figure 32: Particle traces SA solution, vertical cut-plane parallel to the base of the trailer, $0^{\circ}$ yaw. 


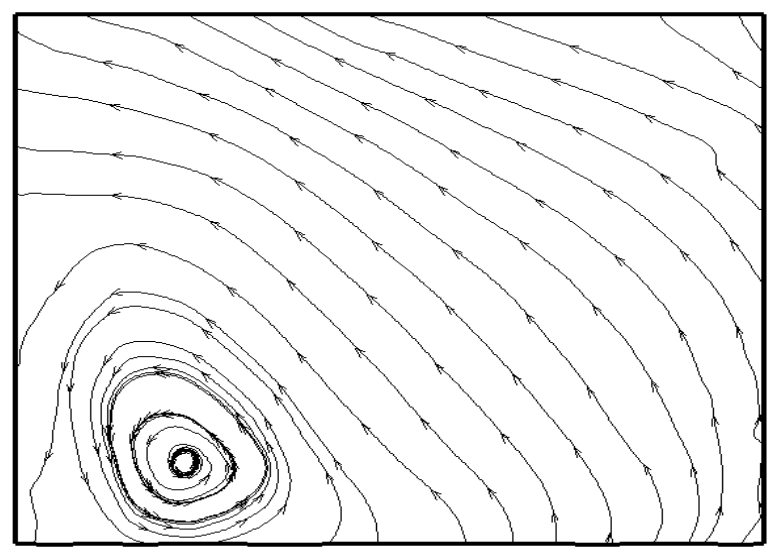

Figure 33: Particle traces time-averaged PIV data, streamwise laser light sheet located at $(\mathrm{y} / \mathrm{w}=0), 0^{\circ}$ yaw.

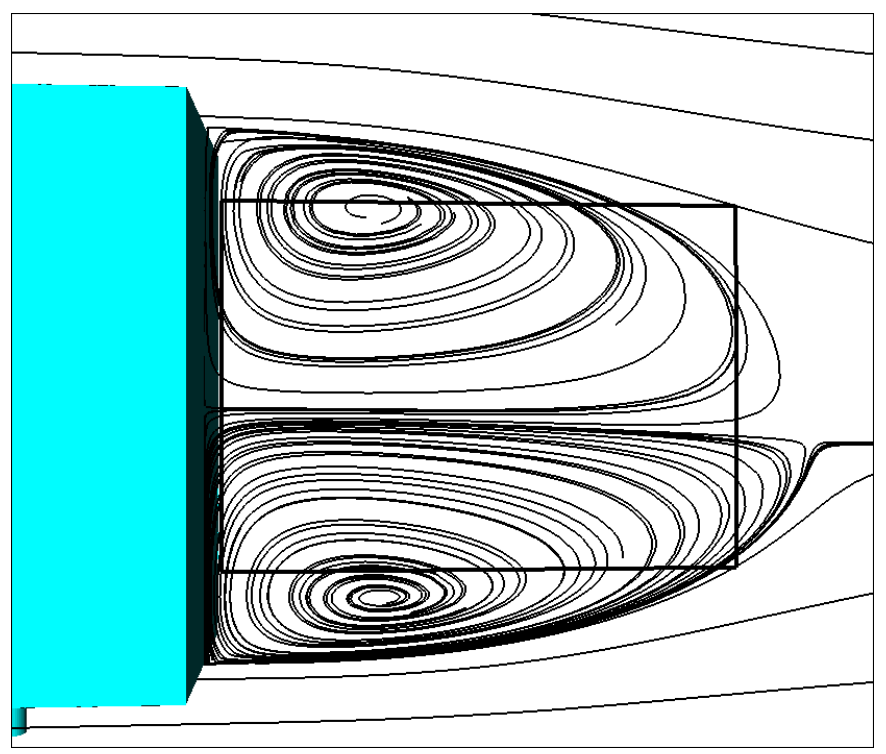

Figure 34: Particle traces SST (BSL) solution, streamwise cut-plane $(\mathrm{y} / \mathrm{w}=0), 0^{\circ}$ yaw. 


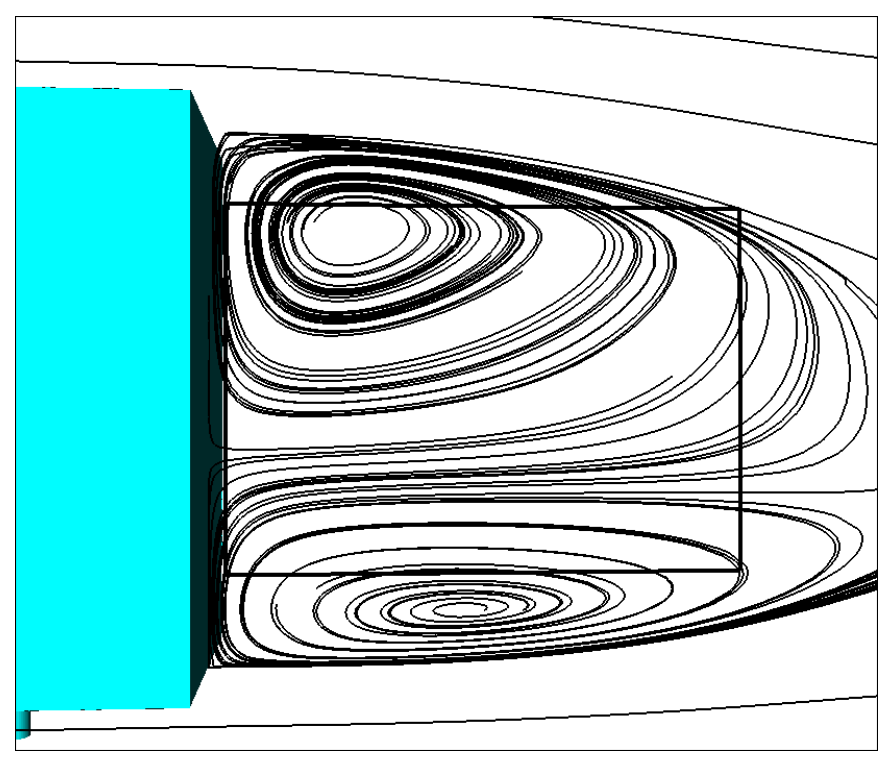

Figure 35: Particle traces Wilcox $k-w$ solution, streamwise cut-plane $(y / w=0), 0^{\circ}$ yaw.

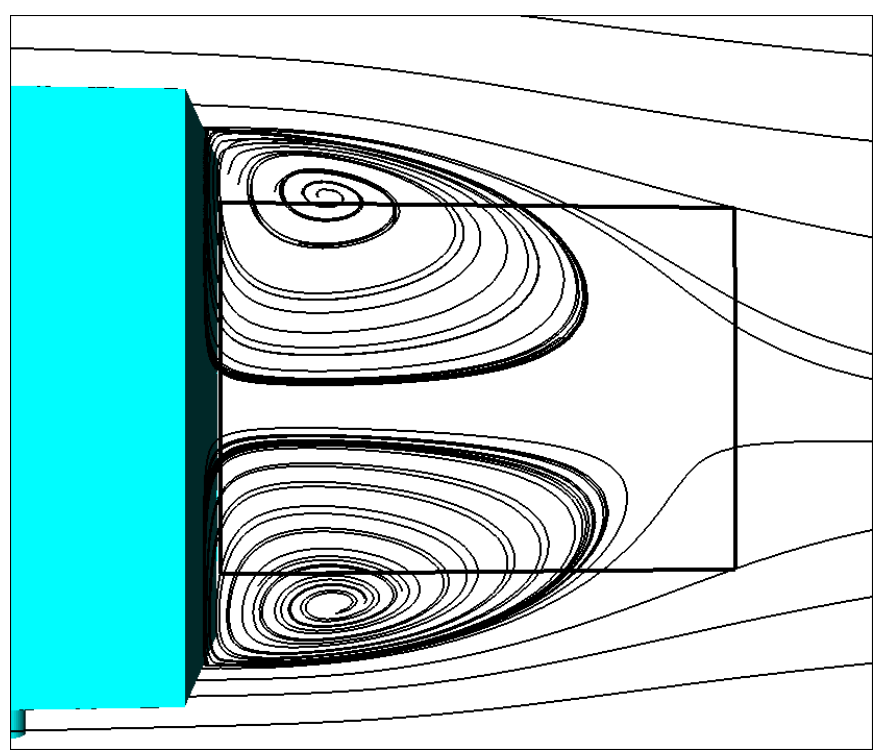

Figure 36: Particle traces SA solution, streamwise plane $(\mathrm{y} / \mathrm{w}=0), 0^{\circ}$ yaw. 


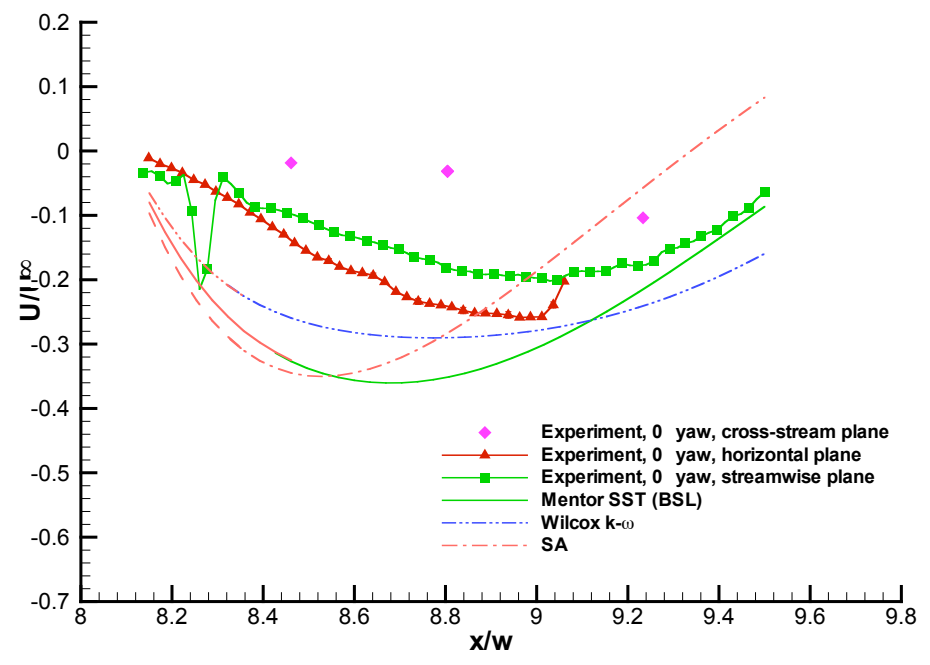

Figure 37: GTS body centerline wake velocities, comparison of computed and PIV data, $0^{\circ}$ yaw.

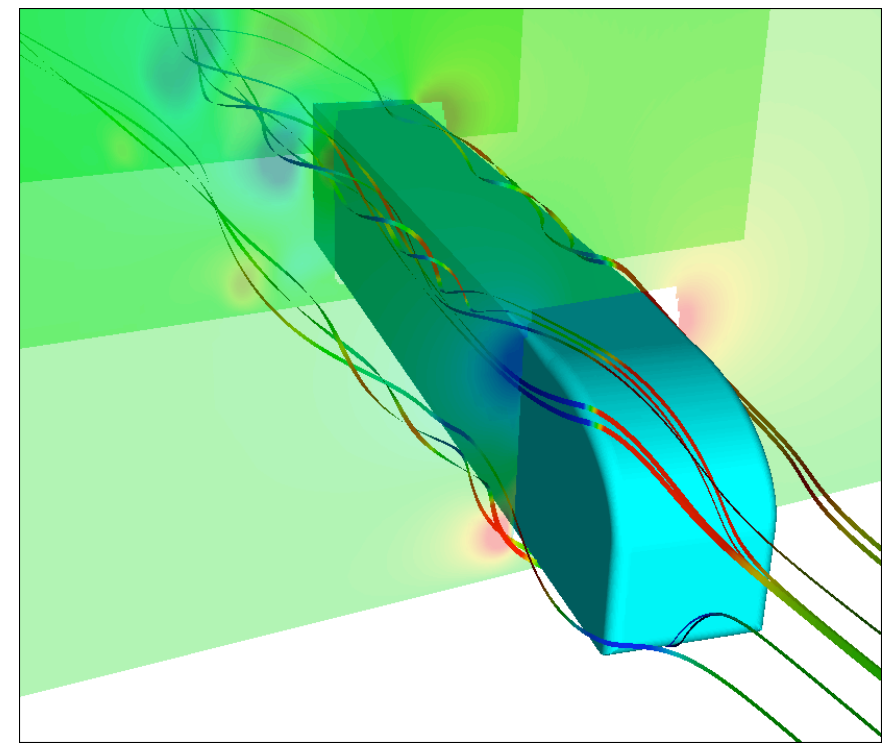

Figure 39: Particle traces SA solution, streamwise plane $(y / w=0), 0^{\circ}$ yaw. 

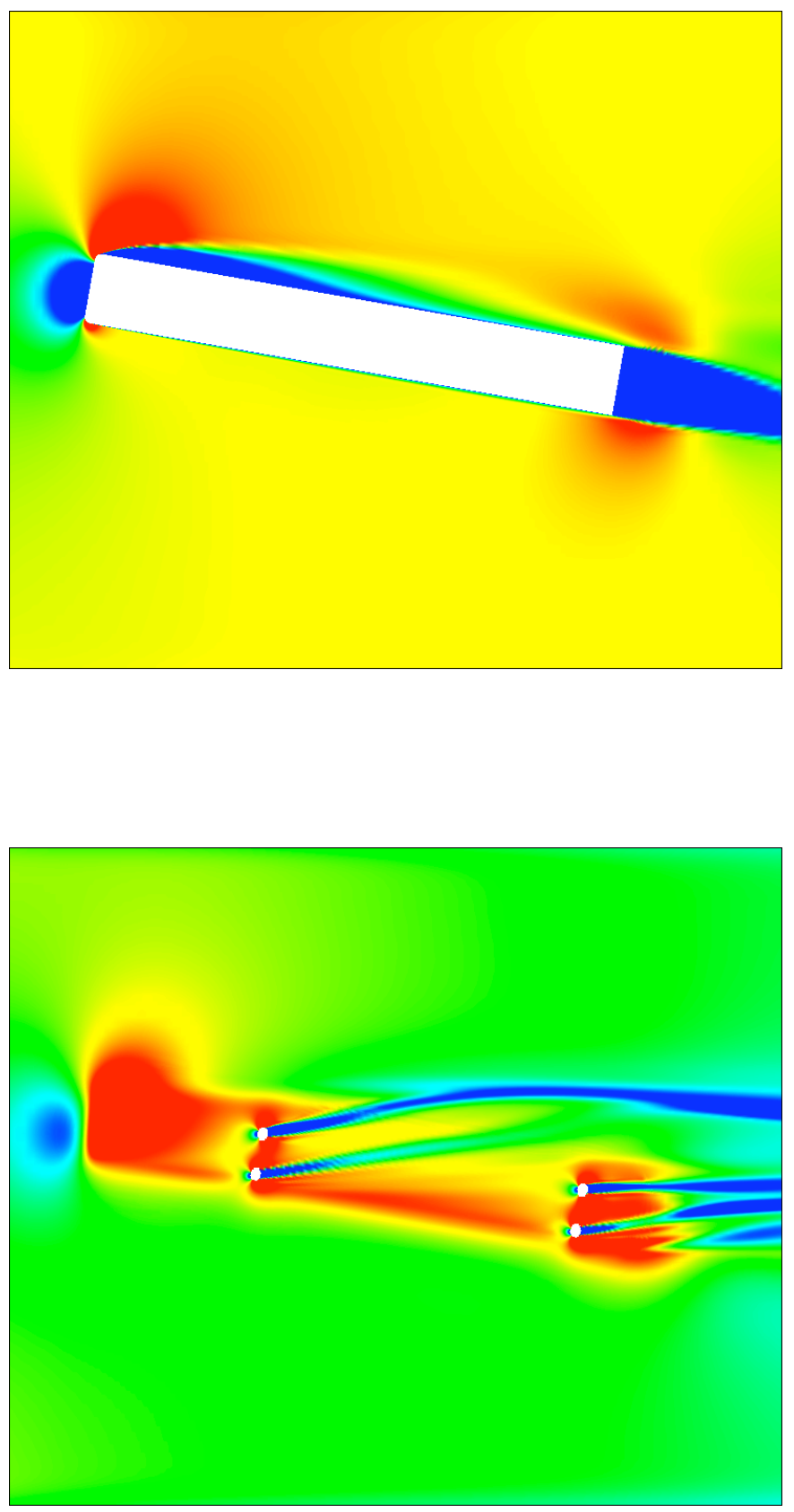

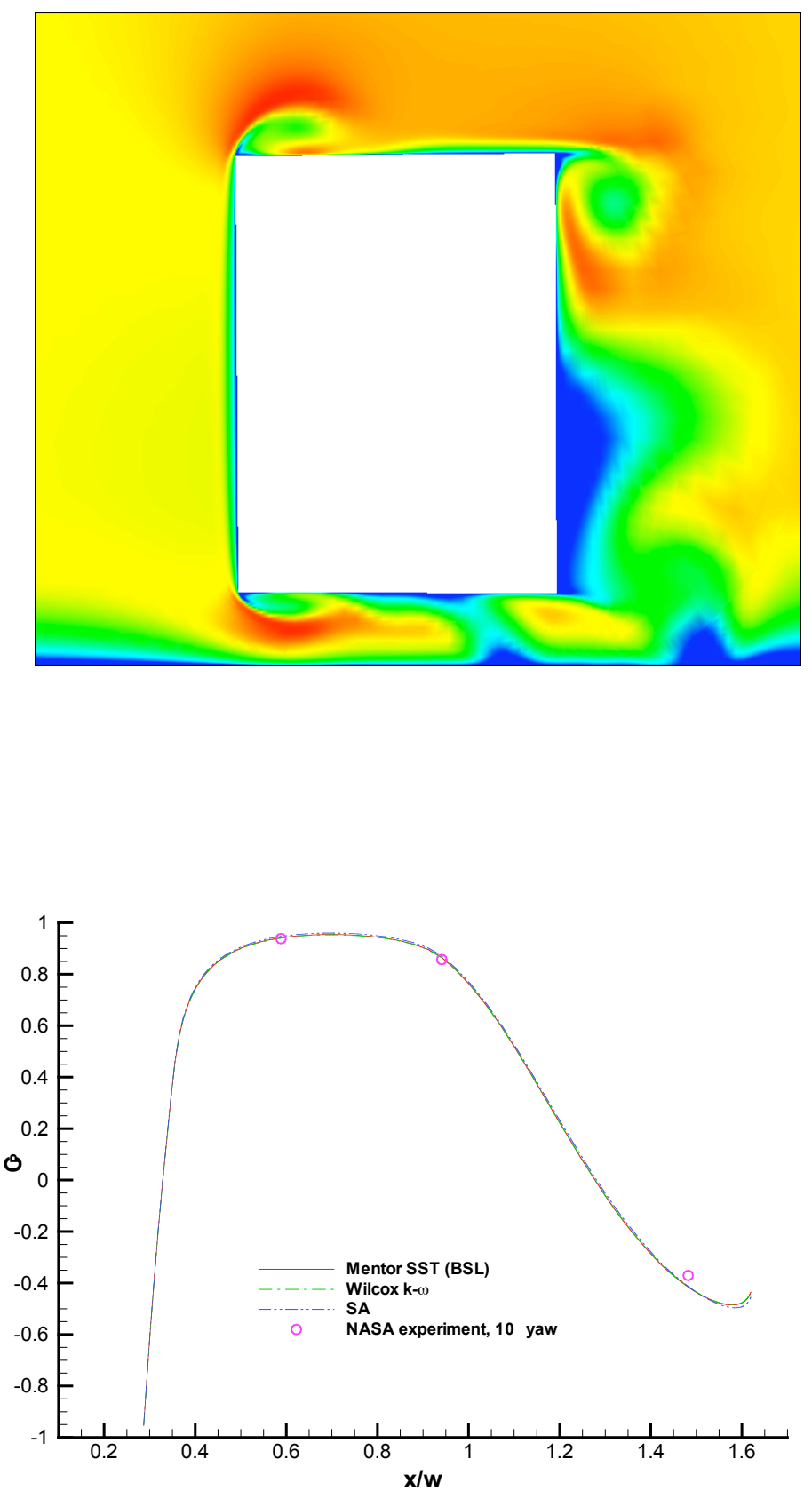

Figure 40: Comparison of the computed $\mathrm{C}_{\mathrm{p}}$ to the NASA experimental data, front of GTS, $10^{\circ}$ yaw. 


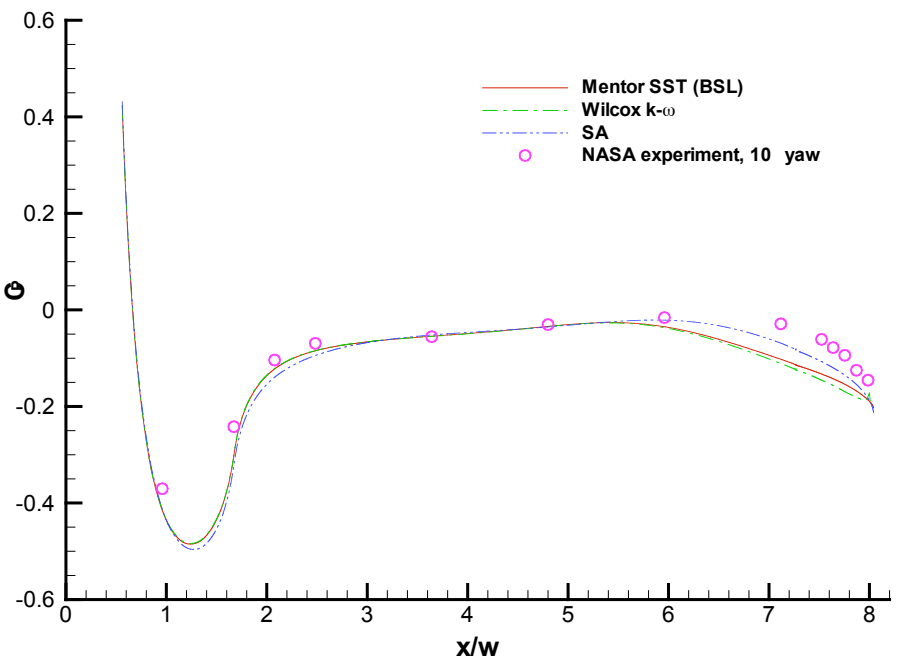

Figure 41: Comparison of the computed $\mathrm{C}_{\mathrm{p}}$ to the NASA experimental data, top of GTS, $10^{\circ}$ yaw.

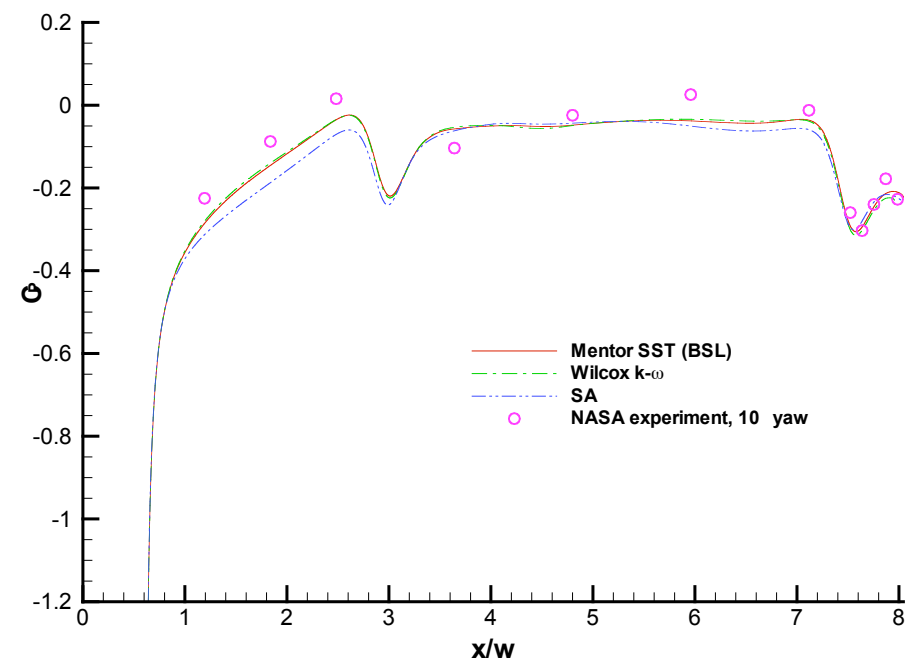

Figure 42: Comparison of the computed $C_{p}$ to the NASA experimental data, bottom of GTS, $10^{\circ}$ yaw. 


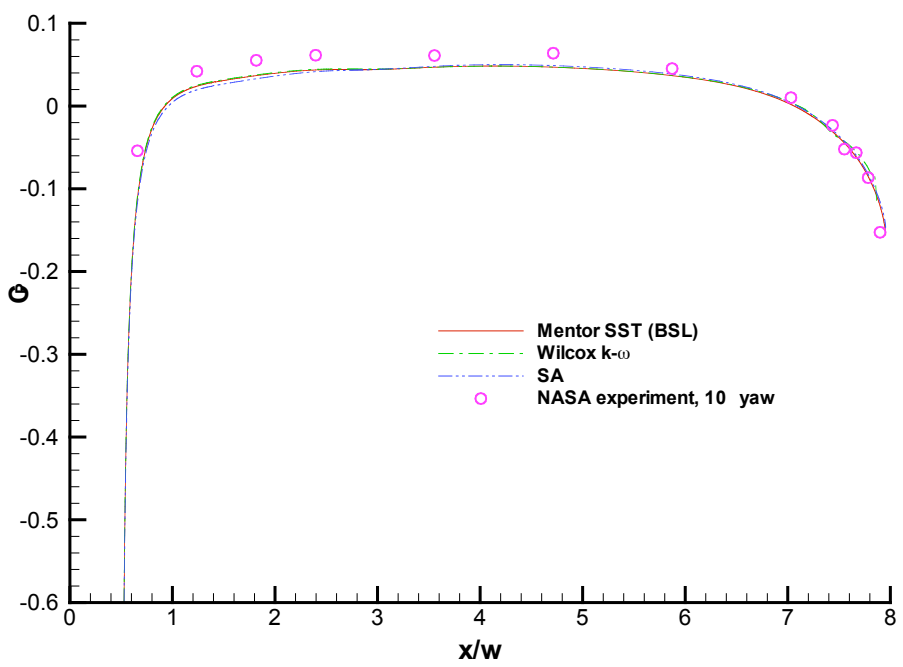

Figure 43: Comparison of the computed $\mathrm{C}_{\mathrm{p}}$ to the NASA experimental data, left side (looking upstream) of GTS, $10^{\circ}$ yaw.

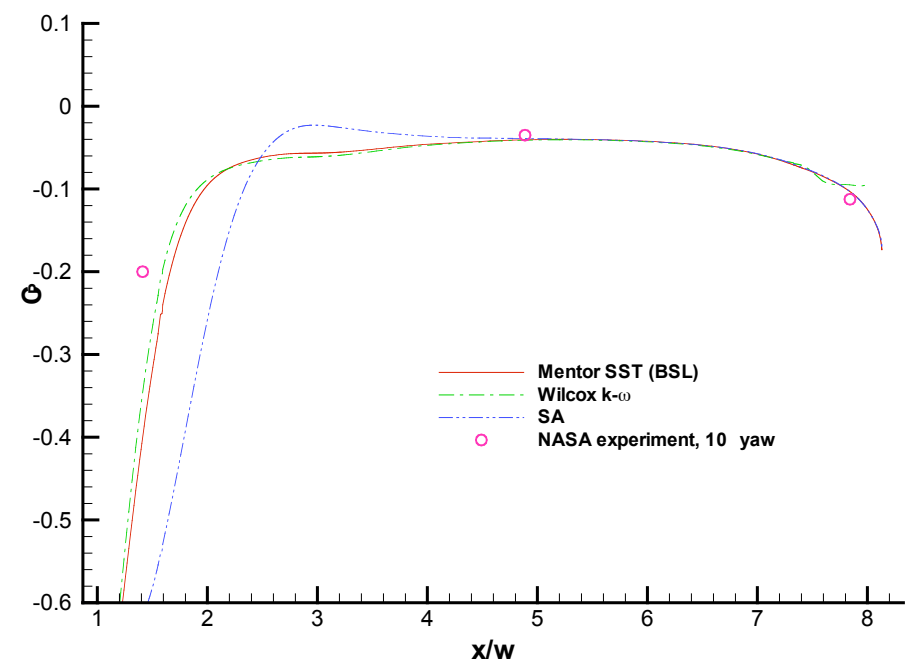

Figure 44: Comparison of the computed $\mathrm{C}_{\mathrm{p}}$ to the NASA experimental data, right side (looking upstream) of GTS, $10^{\circ}$ yaw. 


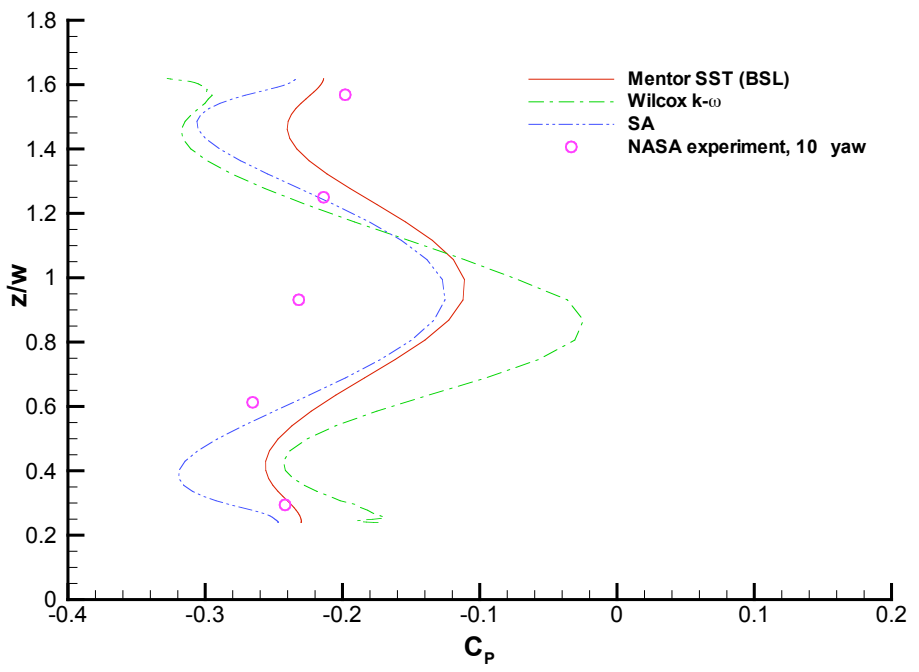

Figure 45: Comparison of the computed $\mathrm{C}_{\mathrm{p}}$ to the NASA experimental data, base of GTS, $10^{\circ}$ yaw.

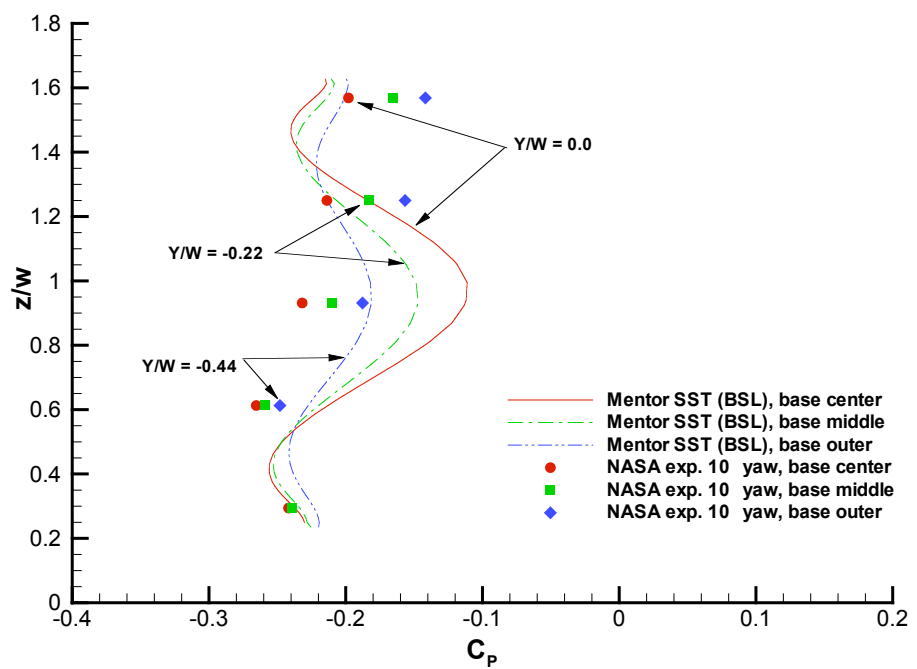

Figure 46: Comparison of the computed $\mathrm{C}_{\mathrm{p}}$ to the NASA experimental data, base of GTS with all pressure taps, $10^{\circ}$ yaw. 


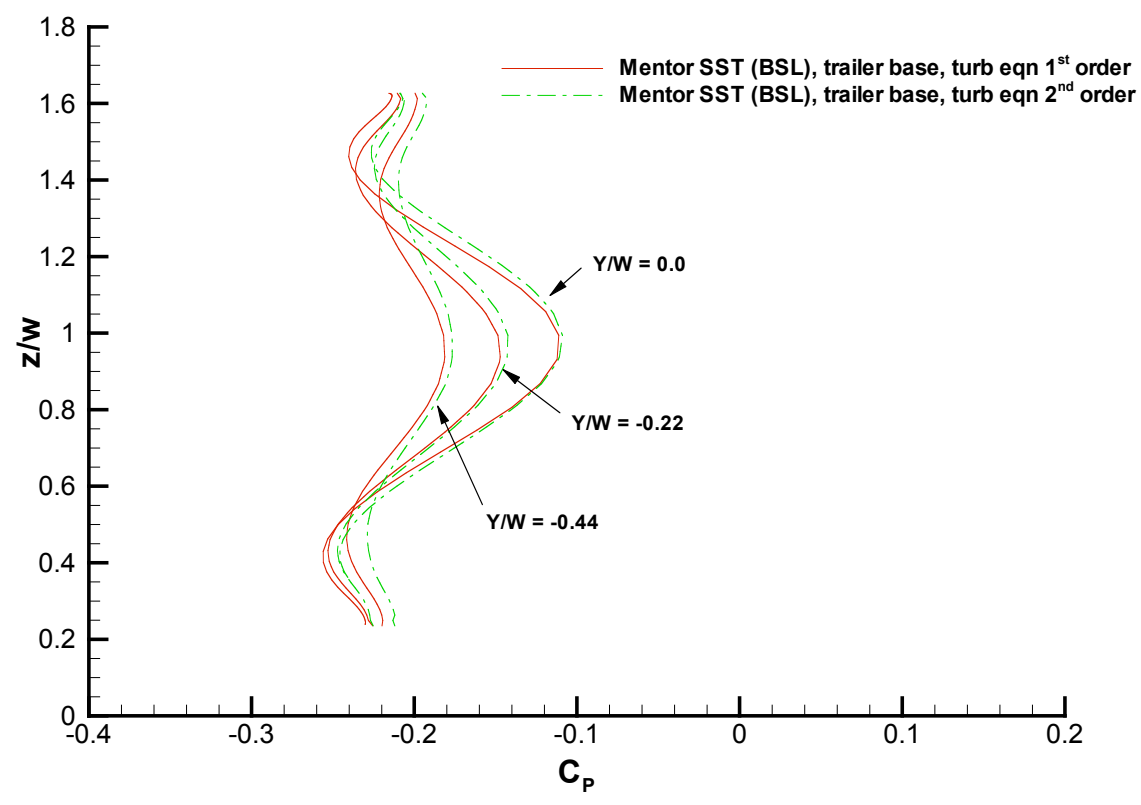

Figure 47: Discretization order study with Mentor SST (BSL) turbulence model, base of GTS, $10^{\circ}$ yaw.

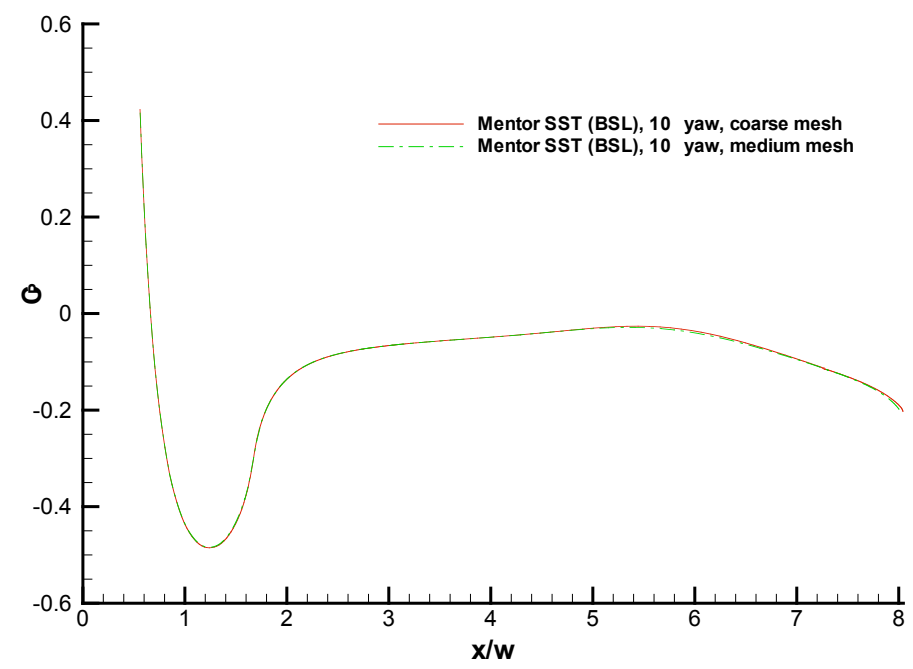

Figure 48: Grid resolution study with Mentor SST (BSL) turbulence model, top of GTS, $10^{\circ}$ yaw. 


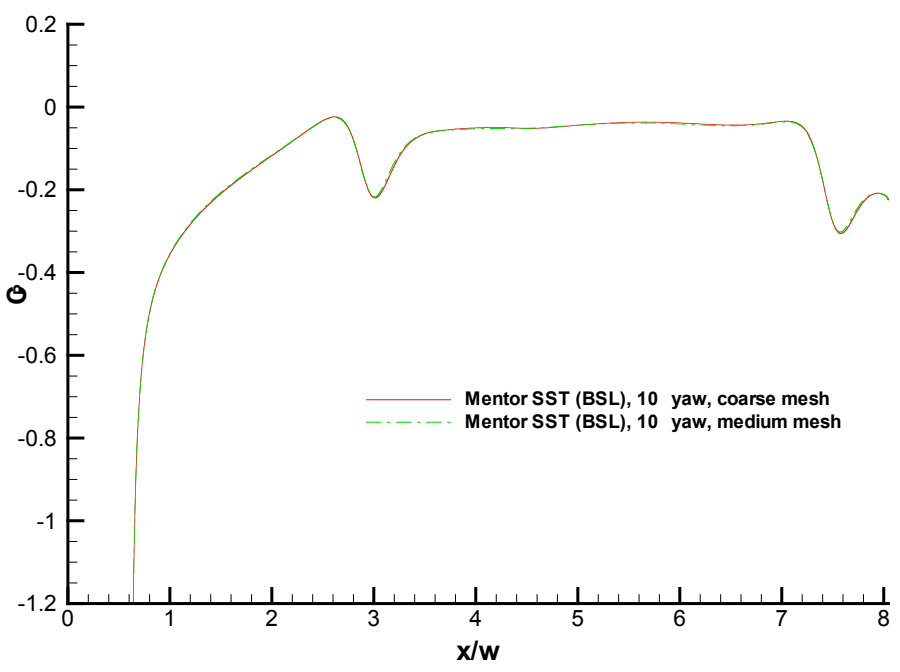

Figure 49: Grid resolution study with Mentor SST (BSL) turbulence model, bottom of GTS, $10^{\circ}$ yaw.

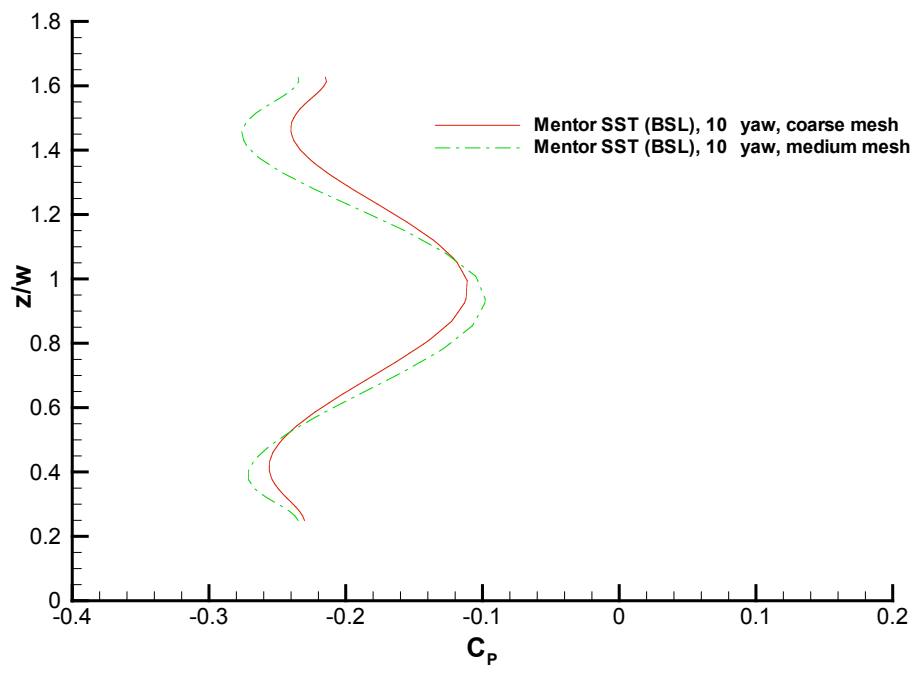

Figure 50: Grid resolution study with Mentor SST (BSL) turbulence model, base of GTS, $10^{\circ}$ yaw. 


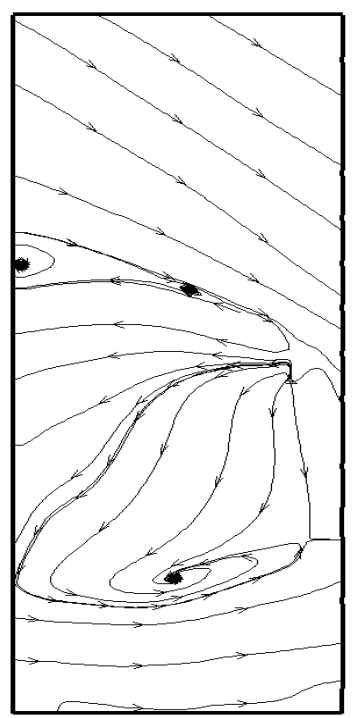

Figure 51: time averaged PIV data, particle traces, horizontal laser light sheet located at trailer mid-height, $10^{\circ}$ yaw.

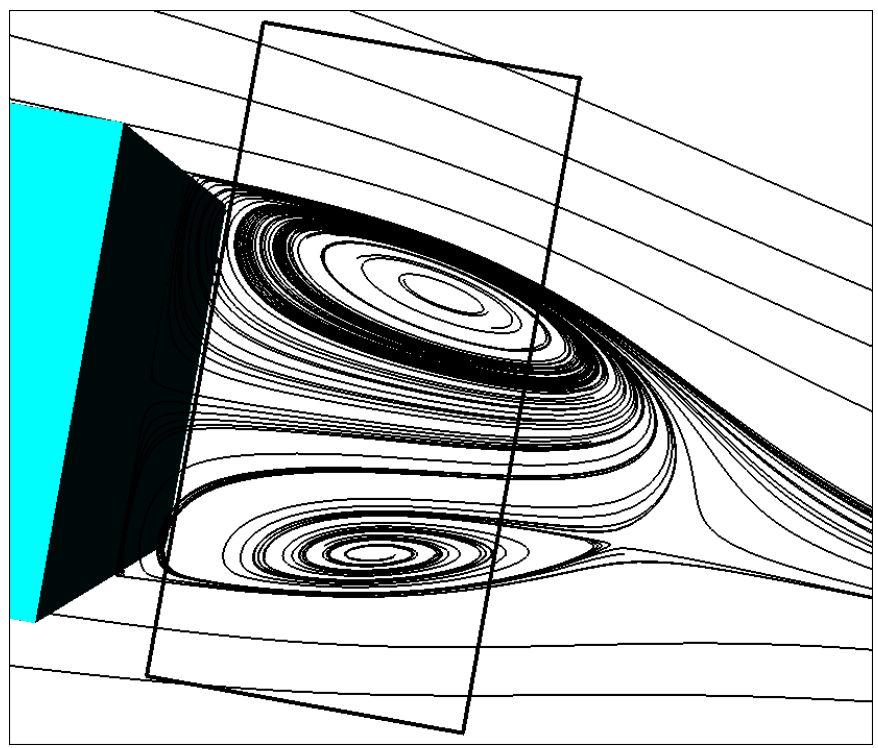

Figure 52: Particle traces SST (BSL) solution, horizontal cut-plane located at trailer mid-height, $10^{\circ}$ yaw. 


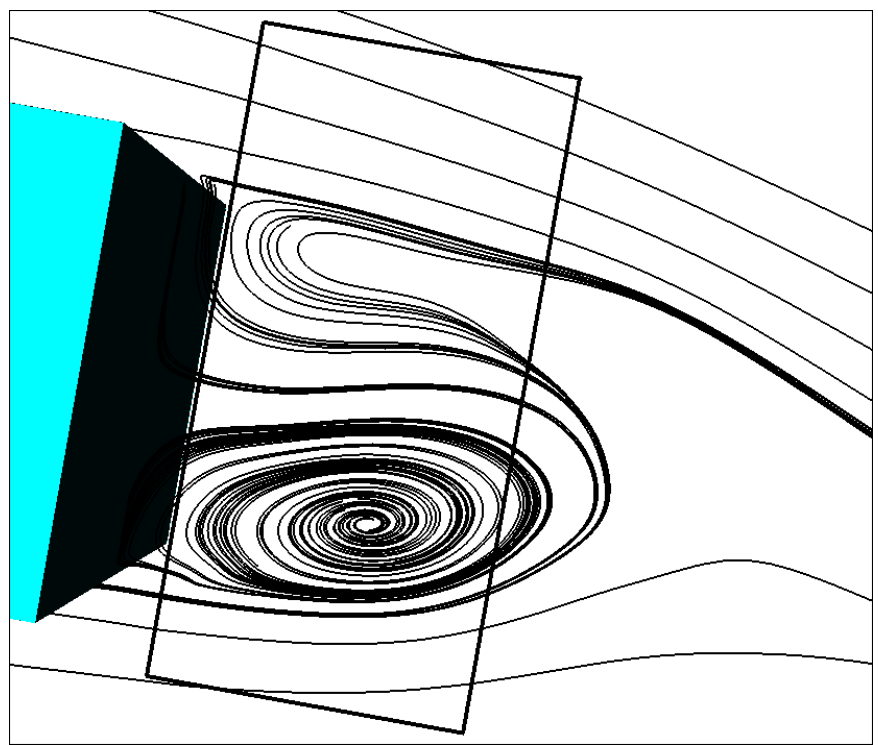

Figure 53: Particle traces KW solution, horizontal cut-plane located at trailer midheight, $10^{\circ}$ yaw.

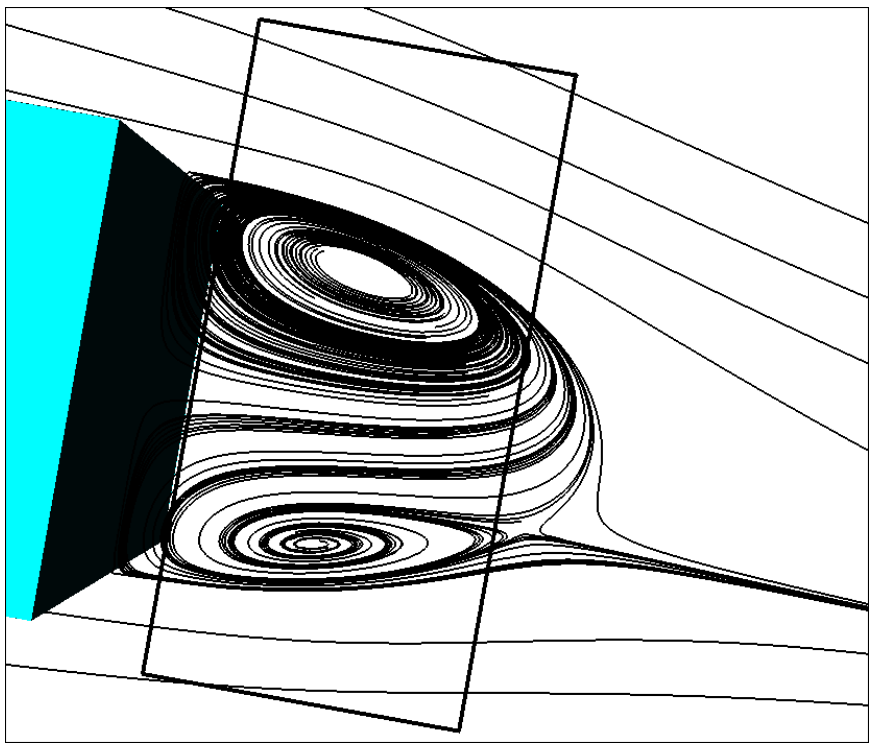

Figure 54: Particle traces SA solution, horizontal cut-plane located at trailer midheight, $10^{\circ}$ yaw. 


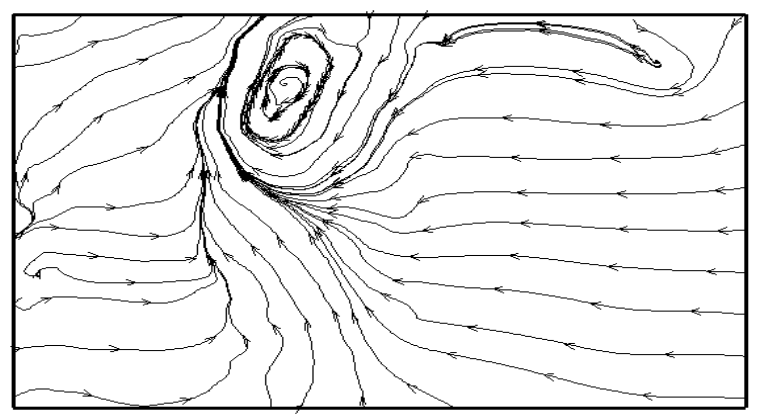

Figure 55: time-averaged PIV data, particle traces, cross-stream laser light sheet located at $\mathrm{x} / \mathrm{w}=$ ???, $10^{\circ}$ yaw.

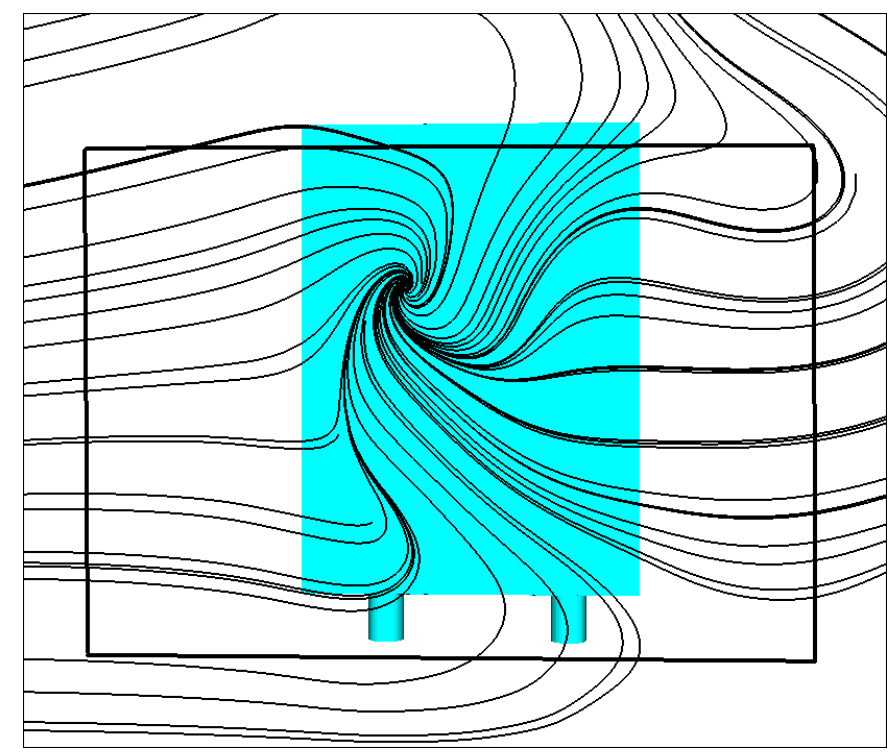

Figure 56: Particle traces SST (BSL) solution, cross-stream cut-plane located at $\mathrm{x} / \mathrm{w}=$ ???, $10^{\circ}$ yaw. 


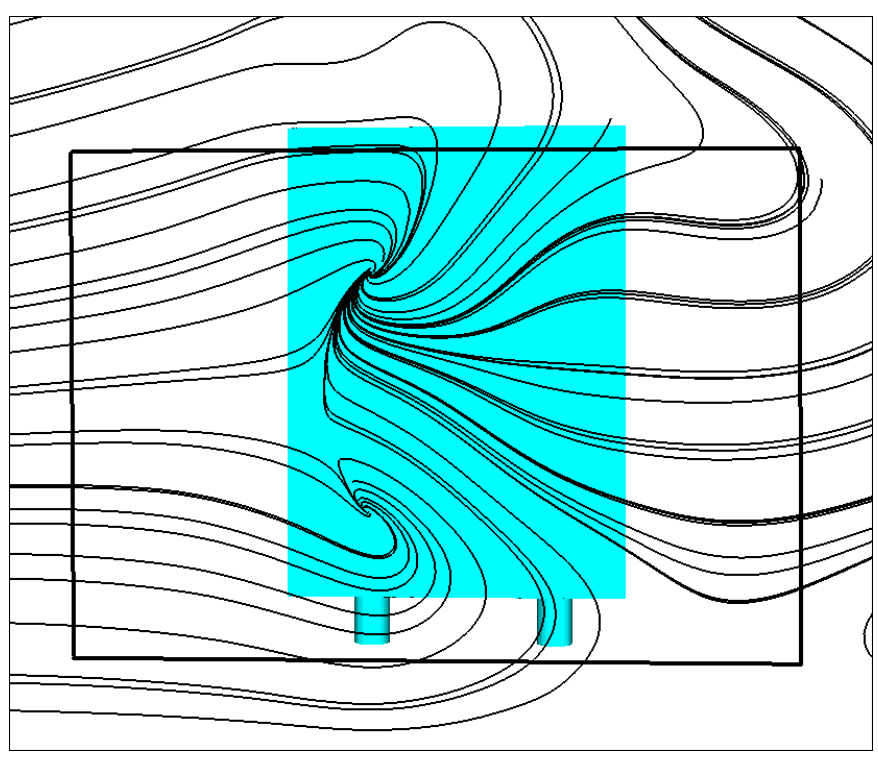

Figure 57: Particle traces KW solution, cross-stream cut-plane located at $\mathrm{x} / \mathrm{w}=$ ???, $10^{\circ}$ yaw.

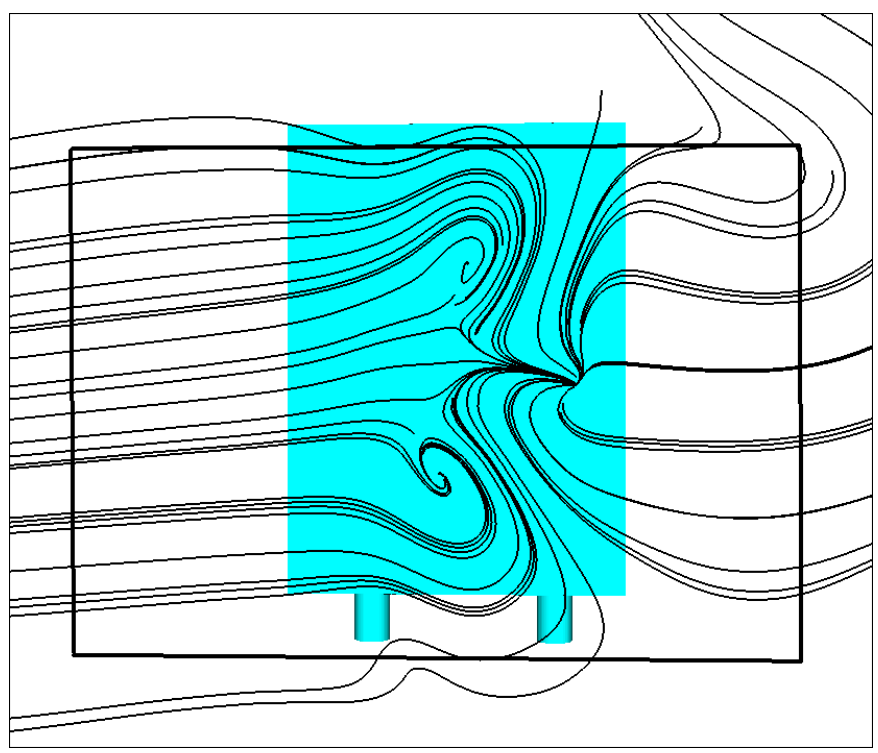

Figure 58: Particle traces SA solution, cross-stream cut-plane located at $\mathrm{x} / \mathrm{w}=$ ???, $10^{\circ}$ yaw. 


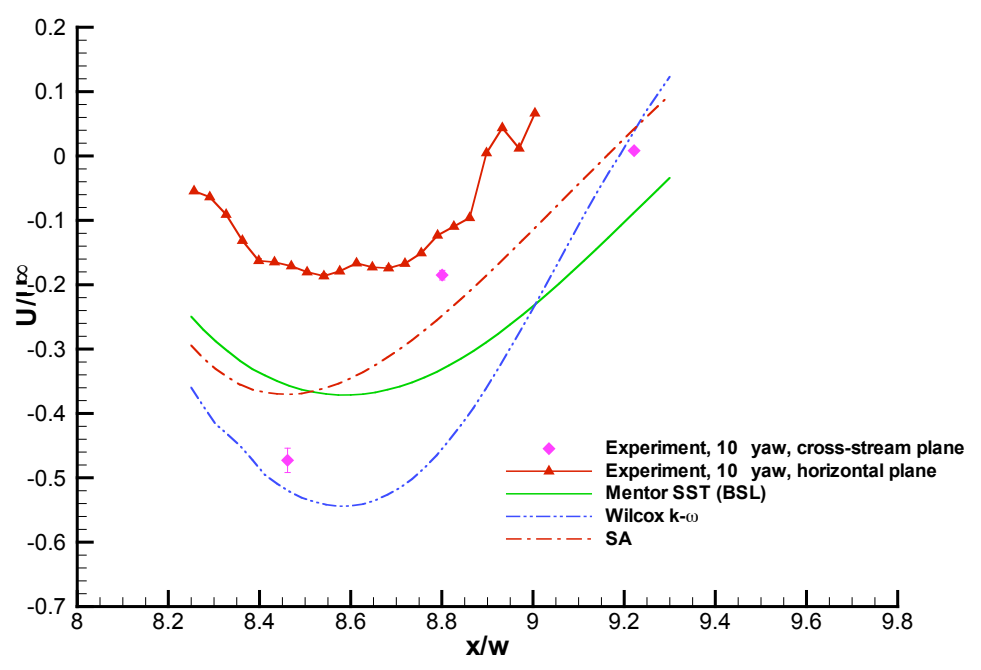

Figure 59: GTS body centerline wake velocities, comparison of computed and PIV data, $10^{\circ}$ yaw.

Figure 60: Oil-Film Interferometry image showing vortex roll-up on top of truck at $10^{\circ}$ deg yaw, ref [3]

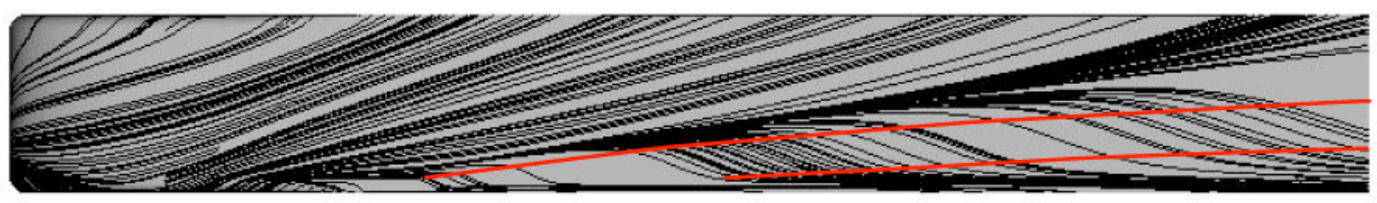

Figure 61: The SST solution, comparing the particle traces confined to the top surface of the trailer highlighting the separation and the reattachment lines to the experimental data.

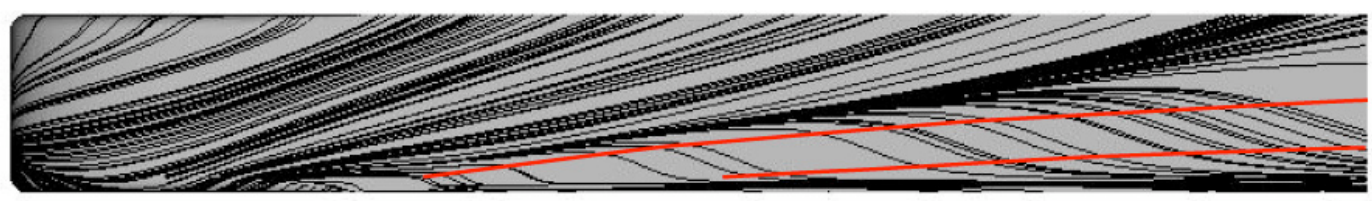


Figure 62: The SST solution, comparing the particle traces confined to the top surface of the trailer highlighting the separation and the reattachment lines to the experimental data.

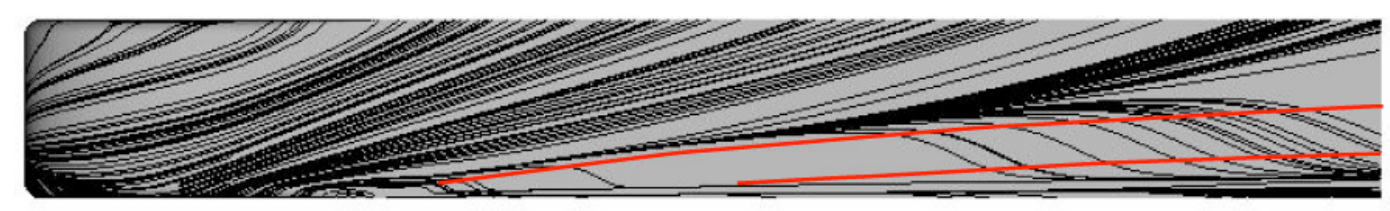

Figure 63: The SA solution, comparing the particle traces confined to the top surface of the trailer highlighting the separation and the reattachment lines to the experimental data.

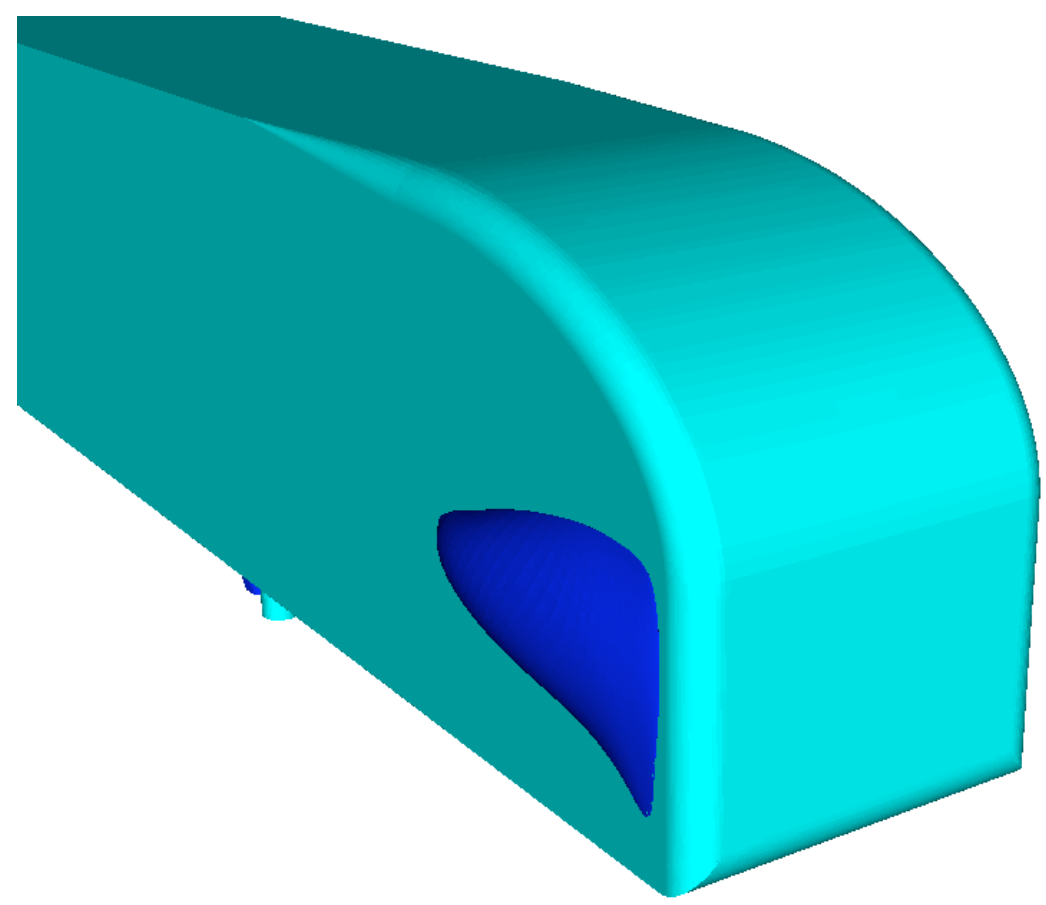

Figure 64: position of the separation bubble in the leeward side of the GTS, SST solution, $10^{\circ}$ yaw. 


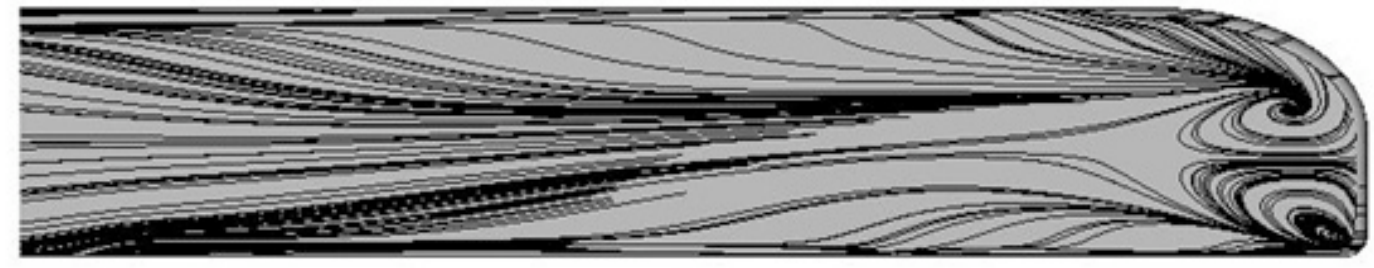

Figure 65: The SST solution, $10^{\circ}$ yaw, particle traces confined to the leeward side of GTS highlighting the separation and the reattachment lines.

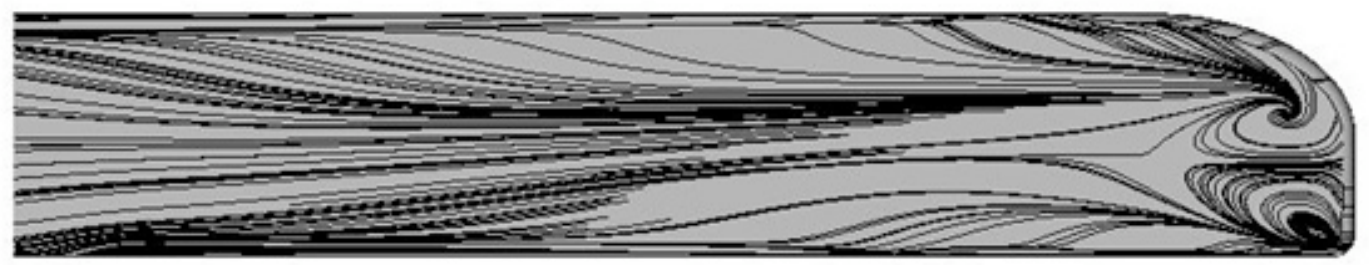

Figure 66: The $\mathrm{KW}$ solution, $10^{\circ}$ yaw, particle traces confined to the leeward side of GTS highlighting the separation and the reattachment lines.

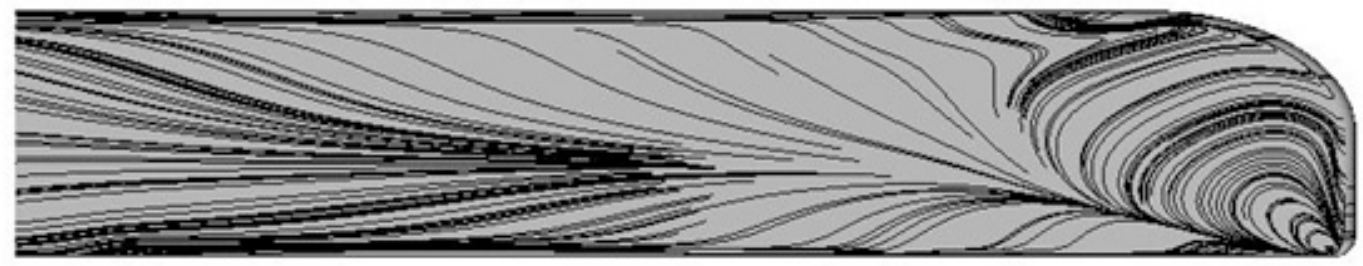

Figure 67: The SA solution, $10^{\circ}$ yaw, particle traces confined to the leeward side of GTS highlighting the separation and the reattachment lines. 


\section{Reference}

${ }^{1}$ Highway Statistics, US. Government Printing Office, SSOP, Washington DC 20402-9328, 1992

${ }^{2}$ Cooper, K.R., “Truck Aerodynamics Reborn—Lessons from the Past,” SAE Paper No. 2003-01-3376, 2003.

${ }^{3}$ Gutierrez, W. T., Hassan, B., Croll, R. H., Rutledge, W. H., "Aerodynamics Overview of the Ground Transportation Systems (GTS) Project for Heavy Vehicle Drag Reduction," SAE Paper No. 960906, Feb. 1996.

${ }^{4}$ Steen, G., "Subsonic Wind Tunnel Test of the Sandia National Laboratories Ground Transportation Vehicle,” TEES Report No. TR-9514, July 1995.

${ }^{5}$ Storms, B., et. al., "An Experimental Study of the Ground Transportation System (GTS) Model in the NASA Ames 7- by 10-Ft Wind Tunnel," NASA/TM-2001-209621, Feb. 2001.

${ }^{6}$ Hassan, B., Gutierrez, W. T., Wolfe, W., Walker, M., "Numerical Prediction of Aerodynamic Drag for Heavy Ground Transportation Vehicle," AIAA Paper AIAA-95-1913, June 1995.

${ }^{7}$ Maddox, S., Squires, K.D., Forsythe, J.R., Detached-Eddy Simulation of the Ground Transportation System, United Engineering Foundation, The Aerodynamics of Heavy Vehicles: Trucks, Buses and Trains, Pacific Grove, California, November, 2002, to be published.

\footnotetext{
${ }^{8}$ Salari, K. and McWherter-Payne, M., "Computational Flow Modeling of a Simplified Integrated Tractor-Trailer Geometry," Sandia Report SAND2003-3383, 2003.
}

${ }^{9}$ Storms, B., NASA Ames Research Center, private communication. 